\title{
PARABOLIC FINITE ELEMENT EQUATIONS IN NONCONVEX POLYGONAL DOMAINS *
}

\author{
P. CHATZIPANTELIDIS ${ }^{1}$, R. D. LAZAROV ${ }^{2, \dagger}$, V. THOMÉE ${ }^{3, \ddagger}$ and L. B. WAHLBIN ${ }^{4, \ddagger}$ \\ ${ }^{1}$ Department of Mathematics, University of Crete, Heraklion, 71409, Greece. \\ email: chatzipa@math.uoc.gr \\ ${ }^{2}$ Department of Mathematics, Texas A\&M University, College Station, TX, 77843, USA. \\ email: lazarov@math.tamu.edu \\ ${ }^{3}$ Department of Mathematics, Chalmers University of Technology and Göteborg University, \\ Göteborg, SE-41296, Sweden. email: thomee@math.chalmers.se \\ ${ }^{4}$ Department of Mathematics, Cornell University, Ithaca, N.Y., 14853, USA. \\ email: wahlbin@math.cornell.edu
}

Dedicated to Björn Engquist on the occasion of his 60th birthday

\begin{abstract}
.
Let $\Omega$ be a bounded nonconvex polygonal domain in the plane. Consider the initial boundary value problem for the heat equation with homogeneous Dirichlet boundary conditions and semidiscrete and fully discrete approximations of its solution by piecewise linear finite elements in space. The purpose of this paper is to show that known results for the stationary, elliptic, case may be carried over to the time dependent parabolic case. A special feature in a polygonal domain is the presence of singularities in the solutions generated by the corners even when the forcing term is smooth. These cause a reduction of the convergence rate in the finite element method unless refinements are employed.
\end{abstract}

AMS subject classification (2000): 65M60, 65N30.

Key words: heat equation, nonconvex polygonal domain, reentrant corner, singularity, semidiscrete finite element method, fully discrete methods, order of convergence, refined meshes.

\section{Introduction.}

The purpose of this paper is to show that certain known error estimates for piecewise linear finite element approximations to solutions of elliptic equations in nonconvex polygonal domains carry over to parabolic problems.

We consider the model initial boundary value problem, for $u=u(x, t)$,

$$
\begin{aligned}
u_{t}-\Delta u=f & \text { in } \Omega, \quad \text { with } u(\cdot, t)=0 \quad \text { on } \partial \Omega, \text { for } t>0, \\
u(\cdot, 0)=v & \text { in } \Omega,
\end{aligned}
$$

*Received 2005. Communicated by XXXXXX.

${ }^{\dagger}$ Partly supported by the U.S. National Science Foundation under Grant DEIA 0218229.

${ }^{\ddagger}$ Partly supported by the U.S. National Science Foundation under Grant DMS 0310539. 
where $\Omega$ is a nonconvex plane polygonal domain. We assume, for simplicity of presentation, that exactly one interior angle $\omega$ is reentrant, i.e., such that $\pi<\omega<2 \pi$. (Since $\omega<2 \pi$, crack problems are not covered.)

The corresponding stationary elliptic problem is the Dirichlet problem

$$
-\Delta u=f \quad \text { in } \Omega, \quad \text { with } u=0 \quad \text { on } \partial \Omega,
$$

and the regularity of the solutions of this problem has been extensively studied, see Grisvard [6], [7]. We set $\beta=\pi / \omega$, and note that $\frac{1}{2}<\beta<1$. In the special case of an L-shaped domain, $\omega=3 \pi / 2$ and $\beta=2 / 3$. Assuming that the associated vertex is at the origin $O$ and describing the domain near $O$ by polar coordinates $(r, \theta)$, with $0<\theta<\omega$, the corner $O$ then gives rise to a singularity in the solution with a leading term near $O$ of the form

$$
\kappa(f) r^{\beta} \sin (\beta \theta)
$$

where, in general, $\kappa(f) \neq 0$ even when $f$ is smooth. A regularity shift-theorem for this problem, shown in Kellogg [8], may be expressed as

$$
\|u\|_{H^{1+s}} \leq C\|f\|_{H^{-1+s}}=C\|\Delta u\|_{H^{-1+s}}, \text { for } 0 \leq s<\beta,
$$

where the $H^{s}=H^{s}(\Omega)$ are fractional order Sobolev spaces, see Section 2 below, but such an estimate with $s \geq \beta$ cannot be expected due to the singularity (1.3). A certain replacement of (1.4) in the limiting case $s=\beta$ was derived by Bacuta, Bramble, and $\mathrm{Xu}[3]$ in a Besov space framework. The singular behavior of the solutions carry over to the parabolic problem (1.1), see [7, Chapter 5].

We now turn to the approximation by the finite element method, using piecewise linear finite element spaces $S_{h} \subset H_{0}^{1}(\Omega)$ based on triangulations $\mathcal{T}_{h}=\{\tau\}$ with $h=\max _{\tau \in \mathcal{T}_{h}} \operatorname{diam}(\tau)$. For simplicity we shall assume throughout that the triangulations are shape-regular, i.e., satisfy a minimum angle condition. By global quasiuniformity we shall mean that there is a constant $c>0$ such that $\min _{\tau \in \mathcal{T}_{h}} \operatorname{diam}(\tau) \geq$ ch.

We begin with the elliptic problem (1.2). The finite element method is then to find $u_{h} \in S_{h}$ such that

$$
\left(\nabla u_{h}, \nabla \chi\right)=(f, \chi), \text { for } \chi \in S_{h} .
$$

For the error in the energy norm in this solution one may show, using the regularity estimate of [3], that

$$
\left\|u_{h}-u\right\|_{H^{1}} \leq C h^{\beta}\|\Delta u\|_{H^{-1+s}}, \text { for } \beta<s \leq 1 \text {. }
$$

By duality one obtains, with $\|\cdot\|=\|\cdot\|_{L_{2}}$, as we shall write for brevity below,

$$
\left\|u_{h}-u\right\| \leq C h^{2 \beta}\|\Delta u\|_{H^{-1+s}}, \text { for } \beta<s \leq 1 .
$$

Error bounds with higher powers of $h$ do not hold in general, even for smooth $f$, because of the lack of regularity of the exact solution caused by the reentrant 
corner. The reduction in the convergence rate from the optimal order $O\left(h^{2}\right)$ in a domain with smooth boundary, or a convex polygonal domain, to $O\left(h^{2 \beta}\right)$ may be avoided by the use of mesh refinement near the corners, cf. Babuška [1] and Raugel [11]. In maximum-norm the global order of convergence is further reduced to essentially $O\left(h^{\beta}\right)$. However, away from the corners the error remains of order $O\left(h^{2 \beta}\right)$. Again, suitable refinements will essentially restore the $O\left(h^{2}\right)$ error bound, cf. Schatz and Wahlbin [14]. These results for the elliptic problem will be reviewed in more detail in Section 2 below.

Our first goal in this work is to see that error estimates of these orders carry over to the semidiscrete parabolic problem, to find $u_{h}(t) \in S_{h}$ for $t \geq 0$ such that

(1.7) $\left(u_{h, t}, \chi\right)+\left(\nabla u_{h}, \nabla \chi\right)=(f, \chi)$, for $\chi \in S_{h}, t>0, \quad$ with $u_{h}(0)=v_{h} \approx v$.

In the error analysis of this problem it is common, following Wheeler [20], to introduce the Ritz projection $R_{h}: H_{0}^{1} \rightarrow S_{h}$ defined by

$$
\left(\nabla R_{h} u, \nabla \chi\right)=(\nabla u, \nabla \chi), \text { for } \chi \in S_{h},
$$

and to write the error as a sum of two terms

$$
u_{h}-u=\left(u_{h}-R_{h} u\right)+\left(R_{h} u-u\right)=\theta+\rho .
$$

The second term then represents the error in an elliptic problem whose exact solution is $u(t)$, and may be bounded in $L_{2}$ by means of the known error bound (1.6). The first term $\theta(t) \in S_{h}$ satisfies the equation

$$
\left(\theta_{t}, \chi\right)+(\nabla \theta, \nabla \chi)=-\left(\rho_{t}, \chi\right), \text { for } \chi \in S_{h}, t>0 .
$$

Introducing the discrete Laplacian $\Delta_{h}: S_{h} \rightarrow S_{h}$ by

$$
-\left(\Delta_{h} \psi, \chi\right)=(\nabla \psi, \nabla \chi), \text { for } \psi, \chi \in S_{h},
$$

the equation (1.9) may be written as

$$
\theta_{t}-\Delta_{h} \theta=-P_{h} \rho_{t}, \quad t>0, \quad \text { with } \theta(0)=v_{h}-R_{h} v,
$$

where $P_{h}$ denotes the orthogonal $L_{2}$ projection onto $S_{h}$. Hence, with $E_{h}(t)=$ $e^{\Delta_{h} t}$ the solution operator of the homogeneous case $f=0$ of (1.7), and assuming for simplicity that $v_{h}=R_{h} v$ so that $\theta(0)=0$, we have

$$
\theta(t)=-\int_{0}^{t} E_{h}(t-\tau) P_{h} \rho_{t}(\tau) d \tau
$$

It is well-known that both $E_{h}(t)$ and $P_{h}$ are stable in $L_{2}$ with norm 1 , and we therefore conclude that the bound for $\rho(t)$ derived from (1.6) yields, with $s \in(\beta, 1]$,

$$
\left\|u_{h}(t)-u(t)\right\| \leq\|\theta(t)\|+\|\rho(t)\| \leq C h^{2 \beta}\left(\|\Delta u(t)\|_{H^{-1+s}}+\int_{0}^{t}\left\|\Delta u_{t}(\tau)\right\|_{H^{-1+s}} d \tau\right) .
$$


It then remains to verify that the solution of (1.1) has enough regularity that the right hand side above is finite under the appropriate assumptions on $v$ and $f$. Such regularity results will normally be expressed below in the form of bounds for the norm of the solution involved in terms of norms of the data. In the present case we use the differential equation in (1.1) to exchange spatial regularity requirements by regularity demands in time, and then show a bound for $u_{t t}$ to demonstrate

$$
\begin{aligned}
& \int_{0}^{t}\left\|\Delta u_{t}(\tau)\right\|_{H^{-1+s}} d \tau \leq \int_{0}^{t}\left(\left\|u_{t t}(\tau)\right\|_{H^{-1+s}}+\left\|f_{t}(\tau)\right\|_{H^{-1+s}}\right) d \tau \\
& \quad \leq C\left(\left\|g_{0}\right\|+\int_{0}^{t}\left\|f_{t}(\tau)\right\| d \tau\right), \quad \text { where } g_{0}=u_{t}(0)=\Delta v+f(0)
\end{aligned}
$$

and a similar estimate for $\|\Delta u(t)\|_{H^{-1+s}}$.

This program is carried out in Section 3 below, and error bounds are also shown for the gradient of the error in $L_{2}$. This section also includes a so-called nonsmooth data estimate for the homogeneous heat equation and a discussion of mesh refinements in the context of the parabolic equation.

Section 4 is devoted to error bounds in maximum-norm. As a preliminary step we show, under the assumption that the family of triangulations underlying the $S_{h}$ is globally quasiuniform, that a resolvent estimate holds in maximum-norm for the discrete Laplacian $-\Delta_{h}$, which, in particular, implies a stability result for the semidiscrete parabolic equation. Again the results from the elliptic case carry over to the parabolic problem so that an essentially $O\left(h^{\beta}\right)$ global error bound can be shown, also for a somewhat weaker assumption on the triangulations than quasi-uniformity, as well as an almost $O\left(h^{2 \beta}\right)$ error bound away from the nonconvex corner. Concluding this section, we show optimal order error bounds in the parabolic case by refining the triangulations, in the same way as for the stationary problem.

In the final Section 5 we give some examples of error bounds for fully discrete methods obtained by discretization in time by finite differences of the spatially semidiscrete parabolic equation.

\section{Review of bounds for the elliptic problem.}

We begin by collecting some known regularity results for the Dirichlet problem (1.2), with particular reference to the situation in a plane polygonal domain with one reentrant corner. This includes a review of some properties of fractional order Sobolev spaces, defined by interpolation. We then recall relevant error estimates, in $L_{2}$ and in maximum-norm, for finite element approximations of (1.2).

Letting $H^{-1}$ denote the dual space of $H_{0}^{1}=H_{0}^{1}(\Omega)$, with duality pairing $\langle\cdot, \cdot\rangle$ over the pivot space $L_{2}$, we define the variational solution of (1.2) for $f \in H^{-1}$ as the function $u \in H_{0}^{1}$ which satisfies

$$
(\nabla u, \nabla \varphi)=\langle f, \varphi\rangle, \quad \forall \varphi \in H_{0}^{1} .
$$


It is well-known that this problem has a unique solution, and that

$$
\|u\|_{H_{0}^{1}}=\|\nabla u\| \leq\|f\|_{H^{-1}} .
$$

For $u \in H_{0}^{1},(2.1)$ defines $f \in H^{-1}$, and then $\Delta: H_{0}^{1} \rightarrow H^{-1}$ by $\Delta u=-f$. In order to discuss further regularity results we shall need to use function spaces with a fractional number of derivatives, cf., e.g., [6], [7]. Letting $H^{m}$ with norm $\|\cdot\|_{H^{m}}$ denote the standard Sobolev spaces of integer order $m \geq 0$, we set, for $s=m+\sigma$, with $0<\sigma<1$,

$$
\|u\|_{H^{s}}=\left(\|u\|_{H^{m}}^{2}+\sum_{|\alpha|=m} \iint_{\Omega \times \Omega} \frac{\left|D^{\alpha} u(x)-D^{\alpha} u(y)\right|^{2}}{|x-y|^{2+2 \sigma}} d x d y\right)^{1 / 2},
$$

and let $H^{s}=H^{s}(\Omega)$ denote the completion of $\mathcal{C}^{\infty}(\bar{\Omega})$ with respect to this norm. Since our $\Omega$ has a Lipschitz boundary there exists a bounded extension operator from $H^{s}(\Omega)$ to $H^{s}\left(\mathbb{R}^{2}\right)$, which is independent of $s$, cf. [7, Section 1.3] for a special such extension operator for a polygonal domain. This may be used to characterize the spaces $H^{s}$ in terms of Fourier transforms in $H^{s}\left(\mathbb{R}^{2}\right)$, and, in turn, to show that, in the terminology of real interpolation theory, see, e.g., Triebel [17], the Sobolev spaces $H^{s}$ have the interpolation property

$$
H^{\theta s_{1}+(1-\theta) s_{2}}=\left[H^{s_{1}}, H^{s_{2}}\right]_{\theta, 2} \text {, for } 0 \leq \theta \leq 1,0 \leq s_{1}<s_{2} .
$$

We shall also have reason to use fractional order spaces with homogeneous boundary conditions, and define

$$
H_{0}^{\sigma}=\left[L_{2}, H_{0}^{1}\right]_{\sigma, 2} \text { and } H_{0}^{1+\sigma}=\left[H_{0}^{1}, H^{2} \cap H_{0}^{1}\right]_{\sigma, 2}, \text { for } 0<\sigma<1,
$$

as well as the negative order spaces

$$
H^{-\sigma}=\left[L_{2}, H^{-1}\right]_{\sigma, 2}, \text { for } 0 \leq \sigma \leq 1 .
$$

We note that, by duality and $(2.2), H^{-\sigma}=\left(H_{0}^{\sigma}\right)^{*}$, for $0 \leq \sigma \leq 1$. We remark that $H_{0}^{1+\sigma}=H^{1+\sigma} \cap H_{0}^{1}$ for $0<\sigma<1$, see [2]. In the statements of several of our error bounds below we shall have reason to know the following fact, showing that $H_{0}^{\sigma}$ does not require any boundary condition for small $\sigma$.

LEMMA 2.1. We have $H_{0}^{\sigma}=H^{\sigma}$ for $0<\sigma<\frac{1}{2}$.

This result was shown in [5] for $\Omega$ with a smooth boundary and stated without proof and in greater generality in [21]. The proof in [5] may be seen to work also for Lipschitz boundaries. For the convenience of the reader we include a proof in an Appendix.

We now collect some known regularity results for the solution of (2.1). Consider first the restriction of $\Delta: H_{0}^{1} \rightarrow H^{-1}$ to $V^{2}=H^{2} \cap H_{0}^{1}$, thus to functions in $H^{2}$ which vanish on $\partial \Omega$. The range of $\Delta$,

$$
R\left(\Delta ; V^{2}\right)=\left\{f \in L_{2}: f=\Delta v \text { for some } v \in V^{2}\right\}
$$

is then a closed proper subspace of $L_{2}$, and hence (1.2) does not always have a solution in $V^{2}$ for $f \in L_{2}$. In fact, referring to [6], [7], we have the following: 
Let $\eta=\eta(r)$ be a smooth cutoff function such that $\eta(r) \equiv 1$ near the nonconvex corner $O$ and such that the support of $\eta$ only meets the two edges emerging from $O$. Then, as is seen by a simple calculation, the singular function

$$
S(r, \theta)=\eta(r) r^{\beta} \sin (\beta \theta)
$$

does not belong to $V^{2}$ but is the solution of the variational problem with right hand side $F=-\Delta S \in C^{\infty}(\bar{\Omega})$, with $F=0$ near $O$. We note that $S \in H_{0}^{1+\sigma}$ for $0<\sigma<\beta$. For, using the $K$-functional, one finds easily

$K\left(t, S ; H_{0}^{1}, H^{2} \cap H_{0}^{1}\right)=\inf _{\psi \in H^{2} \cap H_{0}^{1}}\left(\|S-\psi\|_{H_{0}^{1}}+t\|\psi\|_{H^{2}}\right) \leq C \min \left(t^{\beta}, 1\right)$, for $t>0$,

by choosing $\psi(r, \theta)=\psi(t ; r, \theta)=\zeta(t ; r) S(r, \theta)$, with $\zeta(t ; r)=0$ for $r \leq t,=1$ for $r \geq 2 t$, and $\zeta_{r}^{\prime}(t ; r) \leq C / t$, for $t \leq t_{0}$ small, and $\psi(t ; r, \theta)=0$ for $t \geq t_{0}$. Hence

$$
\|S\|_{H_{0}^{1+\sigma}}^{2}=\int_{0}^{\infty} t^{-2 \sigma-1} K\left(t, S ; H_{0}^{1}, H^{2} \cap H_{0}^{1}\right)^{2} d t<\infty, \text { for } 0<\sigma<\beta .
$$

One also sees that $S \notin H^{1+\sigma}$ for $\sigma \geq \beta$.

One can show that for $f \in L_{2}$ there exists a constant $\kappa(f)$ such that

$$
u-\kappa(f) S \in V^{2} .
$$

In fact, following [7],

$$
N:=R\left(\Delta ; V^{2}\right)^{\perp}=\left\{v \in D\left(\Delta ; L_{2}\right): \Delta v=0 \quad \text { in } \Omega, v=0 \quad \text { on } \partial \Omega\right\},
$$

where $\Delta$ denotes the distributional Laplacian on $L_{2}$. In the case of a domain with smooth boundary, $N$ would equal $\{0\}$. In our case with one reentrant corner, $N$ is one-dimensional and spanned by a function $q$ constructed as follows. Let $S_{*}(r, \theta)=\eta(r) r^{-\beta} \sin (\beta \theta)$ be the so-called dual singular function, let $F_{*}=\Delta S_{*}$ (in the distribution sense), which belongs to $\mathcal{C}^{\infty}(\bar{\Omega})$, and let $w \in H_{0}^{1}$ be the variational solution of

$$
-\Delta w=F_{*} \quad \text { in } \Omega, \quad \text { with } w=0 \quad \text { on } \partial \Omega .
$$

Then $q:=S_{*}+w$. To determine $\kappa(f)$ in (2.4) we apply the Laplacian to it. Then, by $(2.5),(f-\kappa(f) \Delta S, q)=0$, so that

$$
\kappa(f)=c(f, q), \quad \text { where } c=1 /(\Delta S, q),
$$

cf. Maz'ya and Plamenevskii [9].

We note that $S_{*}$, and hence also $q$, belongs to $H^{1-s}$ for $\beta<s<1$. In fact, as above, we have

$$
K\left(t, S_{*} ; L_{2}, H_{0}^{1}\right)=\inf _{\psi \in H_{0}^{1}}\left(\left\|S_{*}-\psi\right\|+t\|\psi\|_{H_{0}^{1}}\right) \leq C \min \left(t^{1-\beta}, 1\right), \text { for } t>0
$$


and hence

$$
\left\|S_{*}\right\|_{H_{0}^{1-s}}^{2}=\int_{0}^{\infty} t^{-2(1-s)-1} K\left(t, S_{*} ; L_{2}, H_{0}^{1}\right)^{2} d t<\infty, \quad \text { if } \beta<s<1 .
$$

One may thus write

$$
u=u_{R}+u_{S}, \quad \text { with } u_{S}=\kappa(f) S=c(f, q) S,
$$

where

$$
\left\|u_{R}\right\|_{H^{2}} \leq C\|f\|
$$

Furthermore, the singular part $u_{S}$ satisfies

$$
\left\|u_{S}\right\|_{H^{1+s_{1}}} \leq\|S\|_{H^{1+s_{1}}}|\kappa(f)| \leq C\|f\|_{H^{-1+s}}, \text { for } 0 \leq s_{1}<\beta<s \leq 1,
$$

since $q \in H_{0}^{1-s}$, but $u_{S} \notin H^{1+s}$ for $s \geq \beta$. The splitting (2.6) is only meaningful if $f \in H^{-1+s}$ with $s>\beta$.

The following shift theorem was shown by Kellogg [8].

Lemma 2.2. The solution $u$ of (2.1) satisfies, for $0 \leq s<\beta$, with $C=C_{s}$,

$$
\|u\|_{H^{1+s}} \leq C\|f\|_{H^{-1+s}}=C\|\Delta u\|_{H^{-1+s}} .
$$

In Bacuta, Bramble, and Xu [3], regularity estimates for elliptic boundary value problems are given for the critical value $s=\beta$ in a Besov space setting, which we will use below. A consequence of their result may be expressed as follows:

LEMMA 2.3. For the solution of (2.1) we have, for $\beta<s \leq 1$, with $C=C_{s}$,

$$
\|u\|_{B_{2}^{1+\beta, \infty}} \leq C_{s}\|f\|_{H^{-1+s}}=C\|\Delta u\|_{H^{-1+s}}, \quad \text { where } B_{2}^{1+\beta, \infty}=\left[H^{1}, H^{2}\right]_{\beta, \infty} .
$$

When we study estimates in maximum-norm below we shall need the following result showing that for certain $f$ the solution of $(2.1)$ is in $\mathcal{C}^{\beta}=\mathcal{C}^{\beta}(\bar{\Omega})$. We denote the norm in $\mathcal{C}^{\beta}$ by $\|\cdot\|_{\mathcal{C}^{\beta}}$.

LEMMA 2.4. Let $u$ be the solution of (2.1). For any $s$ with $\beta<s \leq 1$ we have, with $C=C_{s}$,

$$
\|u\|_{\mathcal{C}^{\beta}} \leq C\|f\|_{H^{-1+s}}=C\|\Delta u\|_{H^{-1+s}} .
$$

Proof. Since $s>\beta$ we may write $u=u_{R}+u_{S}$ as in (2.6). As is easily seen, $S \in \mathcal{C}^{\beta}$, and, using also the second inequality in (2.8), we therefore have

$$
\left\|u_{S}\right\|_{\mathcal{C}^{\beta}} \leq C|\kappa(f)| \leq C\|f\|_{H^{-1+s}}
$$

By Sobolev's embedding theorem we find

$$
\left\|u_{R}\right\|_{\mathcal{C}^{\beta}} \leq C\left\|u_{R}\right\|_{H^{1+s_{0}}}, \quad \text { where, e.g., } s_{0}=(2 \beta+s) / 3 \in(\beta, s) .
$$


To bound the latter norm, recall (2.7). For $s_{1}<\beta$ we have, using Lemma 2.2 for $u$ and (2.8) for $u_{S}$, since $s_{0}>\beta$,

$$
\begin{aligned}
\left\|u_{R}\right\|_{H^{1+s_{1}}} & \leq\|u\|_{H^{1+s_{1}}}+\left\|u_{S}\right\|_{H^{1+s_{1}}} \\
& \leq C\|f\|_{H^{-1+s_{1}}}+C\|f\|_{H^{-1+s_{0}}} \leq C\|f\|_{H^{-1+s_{0}}} .
\end{aligned}
$$

Now choose $\theta \in(0,1)$ so that $s=\theta s_{0}+(1-\theta)$ and then $s_{1}$ so that $s_{0}=$ $\theta s_{1}+(1-\theta)$. Note that this defines $s_{1}<\beta$. By interpolation between (2.7) and (2.11) we now conclude that

$$
\left\|u_{R}\right\|_{H^{1+s_{0}}} \leq C\|f\|_{H^{-1+s}} .
$$

Together with (2.10) and (2.9) this completes the proof.

We consider now the finite element approximation $u_{h}$ of $u$ defined by (1.5). We have the following error estimates exhibiting the orders of convergence attainable with the regularity properties of Lemmas $2.2-2.4$.

Lemma 2.5. We have, with $C=C_{s}$,

$$
\left\|u_{h}-u\right\|_{H^{1}} \leq C h^{\beta}\|\Delta u\|_{H^{-1+s}} \text { for } \beta<s \leq 1 .
$$

Further,

$$
\left\|u_{h}-u\right\| \leq C h^{2 \beta}\|\Delta u\|_{H^{-1+s}}, \text { for } \beta<s \leq 1,
$$

and

$$
\left\|u_{h}-u\right\| \leq C h^{\beta}\|u\|_{H^{1}} .
$$

Proof. The first two estimates are shown in [3], using Lemma 2.3 for (2.12) and a duality argument for (2.13). For the third we also use duality, with

$$
\Delta \psi=\varphi \quad \text { in } \Omega, \quad \psi=0 \quad \text { on } \partial \Omega,
$$

and with $\psi_{h}$ the corresponding finite element solution, to derive, for $\beta<s \leq 1$,

$$
\begin{aligned}
\left|\left(u_{h}-u, \varphi\right)\right| & =\left|\left(\nabla\left(u_{h}-u\right), \nabla\left(\psi-\psi_{h}\right)\right)\right| \leq\left\|\nabla\left(u_{h}-u\right)\right\|\left\|\nabla\left(\psi-\psi_{h}\right)\right\| \\
& \leq C\|\nabla u\| h^{\beta}\|\Delta \psi\|_{H^{-1+s}} \leq C h^{\beta}\|\nabla u\|\|\varphi\|,
\end{aligned}
$$

which shows the desired result.

We now turn to estimates in the maximum-norm, or the norm in $\mathcal{C}=\mathcal{C}(\Omega)$, which we denote $\|\cdot\|_{\mathcal{C}}$. We first show that, under the assumption of quasiuniformity, the global order of convergence is of order essentially $O\left(h^{\beta}\right)$. Here and below we use the notation

$$
\ell_{h}=\max (\log (1 / h), 1) .
$$

Lemma 2.6. Assume that the family of triangulations underlying the family $S_{h}$ is globally quasiuniform. Then we have

$$
\left\|u_{h}-u\right\|_{\mathcal{C}} \leq C h^{\beta} \ell_{h}\|u\|_{\mathcal{C}^{\beta}} .
$$


Proof. With $I_{h}$ the interpolant into $S_{h}$, this follows at once by the almost best approximation property, see Schatz [12],

$$
\left\|u_{h}-u\right\|_{\mathcal{C}} \leq C \ell_{h}\left\|I_{h} u-u\right\|_{\mathcal{C}}
$$

together with a standard approximation result.

We shall now derive a maximum-norm error estimate by using the gradient estimate (2.12). This estimate is marginally weaker in terms of regularity requirements, but uses a weaker assumption on the triangulations than that associated with global quasiuniformity. The proof is based on a discrete Sobolev type inequality, specific to our two-dimensional situation.

LEMmA 2.7. Assume the triangulations are such that $h_{\min } \geq C h^{\gamma}$ for some $\gamma>0$. Then, for any $s, s_{1}$ with $0 \leq s<s_{1}<\beta$, we have, with $\bar{C}=C_{s, s_{1}}$,

$$
\left\|u_{h}-u\right\|_{\mathcal{C}} \leq C h^{s}\|\Delta u\|_{H^{-1+s_{1}}}
$$

Proof. Under the above condition on the triangulations we have the discrete Sobolev type inequality

$$
\|\chi\|_{\mathcal{C}} \leq C \ell_{h}^{1 / 2}\|\nabla \chi\|, \text { for } \chi \in S_{h} .
$$

In fact (cf. [16, Lemma 5.4]), by a standard inverse inequality, using $h_{\min } \geq C h^{\gamma}$, followed by a continuous Sobolev inequality, we find, for any $p \in[2, \infty)$,

$$
\|\chi\|_{\mathcal{C}} \leq C h_{\min }^{-2 / p}\|\chi\|_{L_{p}} \leq C h^{-2 \gamma / p}\|\chi\|_{L_{p}} \leq C h^{-2 \gamma / p} p^{1 / 2}\|\nabla \chi\|,
$$

from which (2.16) follows by taking $p=\ell_{h}$.

We have

$$
\left\|u_{h}-u\right\|_{\mathcal{C}} \leq\left\|u_{h}-I_{h} u\right\|_{\mathcal{C}}+\left\|I_{h} u-u\right\|_{\mathcal{C}} .
$$

Here, by (2.16), (2.14) and Lemma 2.2, we have, with $s<s_{1}<\beta$,

$$
\begin{aligned}
\left\|u_{h}-I_{h} u\right\|_{\mathcal{C}} & \leq C \ell_{h}^{1 / 2}\left\|\nabla\left(u_{h}-u\right)\right\|+C \ell_{h}^{1 / 2}\left\|\nabla\left(u-I_{h} u\right)\right\| \\
& \leq C \ell_{h}^{1 / 2} h^{s_{1}}\|u\|_{H^{1+s_{1}}} \leq C h^{s}\|\Delta u\|_{H^{-1+s_{1}}} .
\end{aligned}
$$

By Sobolev's inequality and Lemma 2.2,

$$
\left\|I_{h} u-u\right\|_{\mathcal{C}} \leq C h^{s}\|u\|_{\mathcal{C}^{s}} \leq C h^{s}\|u\|_{H^{1+s_{1}}} \leq C h^{s}\|\Delta u\|_{H^{-1+s_{1}}}
$$

which shows the result stated.

Away from the corners of the domain the convergence in maximum-norm is of the same order $O\left(h^{2 \beta}\right)$ as in the global $L_{2}$-error estimate. This follows from the following lemma.

LEMMA 2.8. Let $\Omega_{0} \subset \Omega_{1} \subset \Omega$ be such that $\Omega_{1}$ does not contain any corner of $\Omega$ and the distance between $\partial \Omega_{1} \cap \Omega$ and $\partial \Omega_{0} \cap \Omega$ is positive. Assume that triangulations associated with $S_{h}$ are quasiuniform in $\Omega_{1}$. Then, with $C=C_{s}$,

$$
\left\|u_{h}-u\right\|_{\mathcal{C}\left(\Omega_{0}\right)} \leq C h^{2 \beta}\left(\|u\|_{\mathcal{C}^{2 s}\left(\Omega_{1}\right)}+\|\Delta u\|_{H^{-1+s}}\right), \text { for } \beta<s \leq 1 .
$$


Proof. This is a consequence of the following interior estimate, valid up to the interiors of the sides of $\Omega$, see [19, Example 1.10, p. 415], namely

$$
\left\|u_{h}-u\right\|_{\mathcal{C}\left(\Omega_{0}\right)} \leq C \ell_{h}\left\|I_{h} u-u\right\|_{\mathcal{C}\left(\Omega_{1}\right)}+C\left\|u_{h}-u\right\|,
$$

together with Lemma 2.5 and since $h^{2 s} \ell_{h} \leq C h^{2 \beta}$.

We remark that, in the case of a globally quasiuniform mesh, the singularity at the nonconvex corner pollutes the finite element solution everywhere in $\Omega$ and that therefore the $O\left(h^{2 \beta}\right)$ convergence away from the nonconvex corner is best possible (indeed, even in any negative norm), see [18]. However, optimal order $O(h)$ and $O\left(h^{2}\right)$ convergence in $H^{1}$ and $L_{2}$ respectively, may be obtained by systematically refining the triangulations toward the nonconvex corner. Such refinements were first studied in Babuška [1] and Raugel [11].

To be able to state the result needed later, we introduce some notation. Let $d(x)$ denote the distance to the nonconvex corner, and, with $d_{j}=2^{-j}$, let

$$
\Omega_{j}=\left\{x \in \Omega: d_{j} / 2 \leq d(x) \leq d_{j}\right\}, \text { for } j=0, \ldots, J .
$$

Furthermore, let $\Omega_{j}^{\prime}=\Omega_{j-1} \cup \Omega_{j} \cup \Omega_{j+1}$ and $\Omega_{I}=\left\{x \in \Omega: d(x) \leq d_{J} / 2\right\}$. We now choose $J$ such that $d_{J} \approx h^{1 / \beta}$ where $h$ denotes the meshsize in the interior of the domain. Further we choose $\gamma \geq 1 / \beta$ such that, with $\varepsilon$ any small positive number,

$$
h_{j} \leq C h d_{j}^{1-\beta+\varepsilon} \quad \text { and } c h^{\gamma} \leq h_{I} \leq C h^{1 / \beta}, \quad \text { with } c>0,
$$

where $h_{j}$ denotes the maximal meshsize on $\Omega_{j}$. We also assume that the mesh is locally quasiuniform on each $\Omega_{j}^{\prime}$, that $\operatorname{dim} S_{h} \leq C h^{-2}$ and $h_{\min } \geq h^{\gamma}(\gamma \approx 1 / \beta$ for an "economical" refinement). Construction of families of meshes which fulfil these requirements can be found in the references given, and elsewhere. We then have the following result.

LEMMA 2.9. With triangulations as above, satisfying (i), we have

$\left\|u_{h}-u\right\|+h\left\|\nabla\left(u_{h}-u\right)\right\| \leq C h^{1+s}\|\Delta u\|_{H^{-1+s}}=C h^{1+s}\|f\|_{H^{-1+s}}$, for $s=0,1$.

Proof. We start with the $O(h)$ estimate in $H^{1}, s=1$. With $u=u_{R}+u_{S}$ as above, we have

$$
\left\|u_{h}-u\right\|_{H^{1}} \leq\left\|I_{h} u-u\right\|_{H^{1}} \leq\left\|I_{h} u_{R}-u_{R}\right\|_{H^{1}}+\left\|I_{h} u_{S}-u_{S}\right\|_{H^{1}} .
$$

If $f \in L_{2}$, then $u_{R} \in H^{2}$ and $I_{h} u_{R}$ exists. Further, we have by (2.7),

$$
\left\|I_{h} u_{R}-u_{R}\right\|_{H^{1}} \leq C h\left\|u_{R}\right\|_{H^{2}} \leq C h\|f\| .
$$

Since $|\kappa(f)| \leq C\|f\|$, it now remains to show

$$
\left\|I_{h} S-S\right\|_{H^{1}} \leq C h .
$$


With $h_{j}$ the local meshsize on $\Omega_{j}$ one has

$$
\left\|I_{h} S-S\right\|_{H^{1}\left(\Omega_{j}\right)} \leq C h_{j}\|S\|_{H^{2}\left(\Omega_{j}^{\prime}\right)} \leq C h_{j} d_{j}^{\beta-1} \leq C h d_{j}^{\varepsilon},
$$

and

$$
\left\|I_{h} S-S\right\|_{H^{1}\left(\Omega_{I}\right)} \leq\left\|I_{h} S\right\|_{H^{1}\left(\Omega_{I}\right)}+\|S\|_{H^{1}\left(\Omega_{I}\right)} \leq C d_{J}^{\beta} \leq C h,
$$

which implies (2.17) after taking squares and summing.

The result in $H^{1}$ when $s=0$ is trivial. The full result stated now follows by a standard duality argument.

There is a similar theory for refinements which yield an almost $O\left(h^{2}\right)$ error bound in the maximum-norm. To discuss this we first need to note that we are now able to write the solution of (2.1) as a sum of a singular and a regular function, and with the regular part almost twice continuously differentiable at all corners of $\Omega$. More specifically, we want the regular part to be in $W_{p}^{2}$ for large $p$. In [6, Theorem 4.4.3.7], it is shown that any corner of $\Omega$ gives rise to singularities, expressed in terms of polar coordinates centered at the corner under consideration of the form $S_{j m}(r, \theta)=\eta_{j}(r) r^{\beta_{j m}} \sin \left(\beta_{j m} \theta\right)$, with $\beta_{j m}=$ $m \pi / \omega_{j} \in(0,2)$, where $\omega_{j}$ is the interior angle, $m$ is a positive integer, and $\eta_{j}$ a cutoff-function as before. For the reentrant corner we may have $m=1$ or 2 , for convex corners with $\omega_{j} \in\left(\frac{1}{2} \pi, \pi\right)$ only $m=1$ is possible, and for $\omega_{j} \leq \frac{1}{2} \pi$ no such singularity occurs. The solution of (2.1) may then be written, with $\left\{S_{l}\right\}_{l=1}^{M}$ the finitely many singular functions involved,

$$
u=u_{S}+u_{R}, \quad \text { where } u_{S}=\sum_{l=1}^{M} \kappa_{l}(f) S_{l}(x),
$$

where, for any $p<\infty$,

$$
\left\|u_{R}\right\|_{W_{p}^{2}} \leq C_{p}\|f\|_{L_{p}}
$$

The coefficients $\kappa_{l}(f)$ are the solution of a nonsingular linear system of equations involving the $\left(f, q_{l}\right)$ expressing that $\Delta\left(u-u_{S}\right) \perp q_{j}, j=1, \ldots, M$, where the $q_{j}$ are defined in terms of the dual singular functions similarly to $q$ in Section 2 . Since the essential behavior of $q_{j}$ is $r^{-\beta_{j}} \sin \left(\beta_{j} \theta\right)$ we have

$$
\left|\kappa_{l}(f)\right| \leq C_{p}\|f\|_{L_{p}} \text {, for } p>2 /\left(2-\max _{j \leq M} \beta_{j}\right) .
$$

Recall from above that for these bounds to hold for $p=2$, the singular part $u_{S}$ of the solution only needs to contain one term, corresponding to the single reentrant corner, and refinement is only needed there, but for large $p$, also weaker singularities need to be taken into account, and corresponding refinements, suggested by approximation theory, made at other corners.

Using the notation introduced before Lemma 2.9 for each of the corners, with $\beta$ denoting the minimal corresponding $\beta_{j m}$, we then require, with $\varepsilon$ any small positive number,

(ii) $\quad h_{j} \leq C h d_{j}^{1-\beta / 2+\varepsilon} \quad$ and $c h^{\gamma} \leq h_{I} \leq C h^{2 / \beta} \approx d_{J}$, with $\gamma \geq 2 / \beta, c>0$. 
We note that such refinements also satisfy (i) so that the result of Lemma 2.9 remains valid.

LEMMA 2.10. With triangulations as above, this time satisfying (ii) for each of the corners of $\Omega$, we have for the solutions of (1.5) and (2.1), for any $s \in[1,2)$ and $p<\infty$ sufficiently large, with $C=C_{s, p}$,

$$
\left\|u_{h}-u\right\|_{\mathcal{C}} \leq C h^{s}\|\Delta u\|_{L_{p}}=C h^{s}\|f\|_{L_{p}}
$$

The proof of this lemma is implicitly contained in [14]. Since that paper is quite technical and does not account for the regularity needed for $f$, we give some indications of how a thus amended proof would proceed.

First, the kind of local estimate already used in the proof of Lemma 2.8 above shows that (with an $\Omega_{j}$ associated with one corner)

$$
\left\|u_{h}-u\right\|_{\mathcal{C}\left(\Omega_{j}\right)} \leq C \ell_{h}\left\|I_{h} u-u\right\|_{\mathcal{C}\left(\Omega_{j}^{\prime}\right)}+C d_{j}^{-1}\left\|u_{h}-u\right\|_{L_{2}\left(\Omega_{j}^{\prime}\right)} .
$$

For the first term we have

$$
\left\|I_{h} u-u\right\|_{\mathcal{C}\left(\Omega_{j}^{\prime}\right)} \leq C h_{j}^{2-2 / p}\|u\|_{W_{p}^{2}\left(\Omega_{j, h}^{\prime}\right)},
$$

where $\Omega_{j, h}^{\prime}$ is the smallest meshdomain containing $\Omega_{j}^{\prime}$. Here (noting that only the $S_{l}$ corresponding to the corner under consideration enter),

$$
\|u\|_{W_{p}^{2}\left(\Omega_{j, h}^{\prime}\right)} \leq\left\|u_{R}\right\|_{W_{p}^{2}\left(\Omega_{j, h}^{\prime}\right)}+\sum_{l=1}^{M}\left|\kappa_{l}(f)\right|\left\|S_{l}\right\|_{W_{p}^{2}\left(\Omega_{j, h}^{\prime}\right)} \leq C_{p} d_{j}^{\beta-2+2 / p}\|f\|_{L_{p}},
$$

where we have used (2.18) and $\left\|S_{l}\right\|_{W_{p}^{2}\left(\Omega_{j, h}^{\prime}\right)} \leq C d_{j}^{\beta-2+2 / p}$. Hence, with (ii),

$$
\left\|I_{h} u-u\right\|_{\mathcal{C}\left(\Omega_{j}^{\prime}\right)} \leq C_{p} h_{j}^{2-2 / p} d_{j}^{\beta-2+2 / p}\|f\|_{L_{p}} \leq C_{p} h^{2-2 / p}\|f\|_{L_{p}} .
$$

On the innermost domain $\Omega_{I}$, using the discrete Sobolev inequality,

$$
\begin{aligned}
& \left\|u_{h}-u\right\|_{\mathcal{C}\left(\Omega_{I}\right)} \leq\left\|I_{h} u-u\right\|_{\mathcal{C}\left(\Omega_{I}\right)}+\left\|I_{h} u-u_{h}\right\|_{\mathcal{C}\left(\Omega_{I}\right)} \\
& \quad \leq\left\|I_{h} u-u\right\|_{\mathcal{C}\left(\Omega_{I}\right)}+C \ell_{h}^{1 / 2}\left\|I_{h} u-u_{h}\right\|_{H^{1}\left(\Omega_{I}\right)} \\
& \quad \leq\left\|I_{h} u-u\right\|_{\mathcal{C}\left(\Omega_{I}\right)}+C \ell_{h}^{1 / 2}\left\|u_{h}-u\right\|_{H^{1}\left(\Omega_{I}\right)}+C \ell_{h}^{1 / 2}\left\|I_{h} u-u\right\|_{H^{1}\left(\Omega_{I}\right)} .
\end{aligned}
$$

The two terms involving the interpolation error are easily bounded in the desired way. For the middle term we now have the local estimate (known to be valid also at the corner)

$$
\left\|u_{h}-u\right\|_{H^{1}\left(\Omega_{I}\right)} \leq C\left\|I_{h} u-u\right\|_{H^{1}\left(\Omega_{I}^{\prime}\right)}+C d_{I}^{-1}\left\|u_{h}-u\right\|_{L_{2}\left(\Omega_{I}^{\prime}\right)} .
$$

Again the first term on the right is easily handled.

To complete the proof one still has to treat the terms $d_{j}^{-1}\left\|u_{h}-u\right\|_{L_{2}\left(\Omega_{j}^{\prime}\right)}$ occurring above. This is the central technicality in [14] and uses a careful duality technique involving a kick-back argument. The development there only needs slight adjustments to account for $\|f\|_{L_{p}}$. 


\section{$3 \quad L_{2}$-based error estimates.}

In this section we show how some of the error estimates in $L_{2}$ for the finite element approximations of the elliptic problem (1.2) may be carried over to the spatially semidiscrete finite element approximation (1.7) of the parabolic problem (1.1). This problem will be considered in this section in the framework of the spatial space $L_{2}$, and we will therefore now restrict the domain of $\Delta$ : $H_{0}^{1} \rightarrow H^{-1}$ to

$$
D(\Delta)=\left\{v \in H_{0}^{1}: \Delta v \in L_{2}\right\}
$$

We note that $-\Delta$ is then a closed densely defined positive definite operator on $L_{2}$, and that $D(\Delta)$ is an extension of the domain $V^{2}$ considered in Section 2 . The functions in $D(\Delta)$ are thus not required to have individual second order derivatives in $L_{2}$. For instance, the singular function $S$ in (2.3) belongs to $D(\Delta)$ but not to $V^{2}$.

In discussing the regularity of the solutions of (1.1) it will be convenient to use certain Hilbert spaces $\dot{H}^{s}$ based on the orthonormal eigenfunctions $\left\{\varphi_{j}\right\}_{j=1}^{\infty}$ and the corresponding eigenvalues $\left\{\lambda_{j}\right\}_{j=1}^{\infty}$ of $-\Delta$. Let thus $\dot{H}^{s}$ be defined by the norm

$$
\|v\|_{\dot{H}^{s}}=\left(\sum_{j=1}^{\infty} \lambda_{j}^{s}\left\langle v, \varphi_{j}\right\rangle^{2}\right)^{1 / 2}, \text { for } s \geq-1, \quad v \in H^{-1}
$$

Then, since both $\dot{H}^{-s}$ and $H^{-s}$ is the uniquely defined interpolation space between $L_{2}$ and $H^{-1}$, we have $\dot{H}^{-s}=H^{-s}$, for $0<s<1$. Also, $\dot{H}^{s}=H_{0}^{s}$ for $0 \leq s \leq 1$, and for $1 \leq s \leq 2, \dot{H}^{s}$ consists of the functions $u \in H_{0}^{1}$ such that $\Delta u$ is in the negative space $H^{s}-2$, cf. [2]. In particular, (3.1) shows that $D(\Delta)=\dot{H}^{2}$ and the range of $\Delta$ is $L_{2}$. Since obviously $-\Delta$ is an isomorphism between $\dot{H}^{1+s}$ and $\dot{H}^{-1+s}$, the result of Lemma 2.2 may be expressed as

$$
\|u\|_{H^{1+s}} \leq C\|\Delta u\|_{H^{-1+s}} \leq C\|\Delta u\|_{\dot{H}^{-1+s}}=C\|u\|_{\dot{H}^{1+s}}, \text { for } 0 \leq s<\beta .
$$

The solution operator of the homogeneous case $(f=0)$ of $(1.1)$ may be defined as

$$
E(t) v=\sum_{j=1}^{\infty} e^{-\lambda_{j} t}\left\langle v, \varphi_{j}\right\rangle \varphi_{j}, \text { for } v \in H^{-1},
$$

and it follows at once by Parseval's relation that $E(t)$ is a contraction in $L_{2}$ and has the smoothing property

$$
\|E(t) v\|_{\dot{H}^{s_{2}}} \leq C t^{-\left(s_{2}-s_{1}\right) / 2}\|v\|_{\dot{H}^{s_{1}}}, \text { for }-1 \leq s_{1} \leq s_{2} .
$$

In particular, $E(t)$ is an analytic semigroup in $L_{2}$, and $\Delta$ is its generator, cf. Pazy [10].

In the present case of a nonconvex domain, one may loose uniqueness if one allows a solution concept which is too weak. Following [7, Section 5.1.2], consider 
the function $q \in \stackrel{\circ}{W}_{p}^{1}, p<2 /(1+\beta)<2$ defined in the paragraph following (2.5) above. Regard this as solution of (1.1) with $f=0$ and $v=q$ which is constant in time. The semigroup solution $E(t) q$ is different.

For the inhomogeneous problem (1.1), with $v \in L_{2}, f \in L_{1}\left(0, T ; L_{2}\right)$, we define the mild solution by Duhamel's principle,

$$
u(t)=E(t) v+\int_{0}^{t} E(t-\tau) f(\tau) d \tau .
$$

This is unique among so-called "weak" solutions of (1.1) in the class of functions $u \in L_{2}\left(0, T ; H_{0}^{1}\right)$ with $u_{t} \in L_{2}\left(0, T ; H^{-1}\right)$. As remarked in [7, Remark 5.1.2], this class is too weak to allow a meaningful splitting into regular and singular parts. Instead, he considers $v \in H_{0}^{1}, f \in L_{2}\left(0, T ; L_{2}\right)$ in which case the mild solution is unique among "classical" solutions $u \in L_{2}(0, T ; D(\Delta))$, with $u_{t} \in L_{2}\left(0, T ; L_{2}\right)$.

In our regularity results below, we shall make stronger assumptions on $v$ and $f$ which, by (3.4) lead to results needed on $u$, in particular to apply the elliptic theory. In the following result we use the notation

$$
g_{0}=u_{t}(0)=\Delta v+f(0) \text {, for } v \in D(\Delta), f(0) \in L_{2} .
$$

Note that $v \in D(\Delta)$ contains the compatibility condition $v=0$ on $\partial \Omega$ between initial data and the boundary condition in (1.1).

Our first regularity estimates for the solution of (1.1) are as follows.

LEMMA 3.1. Let $u(t)$ be the solution of (1.1), and let $g_{0}$ be defined by (3.5). Then we have, for $0 \leq s<1$ and $t \leq T$, with $C=C_{s, T}$,

$$
\int_{0}^{t}\left(\left\|u_{t}(\tau)\right\|_{\dot{H}^{1+s}}+\left\|u_{t t}(\tau)\right\|_{\dot{H}^{-1+s}}\right) d \tau \leq C\left(\left\|g_{0}\right\|+\int_{0}^{t}\left\|f_{t}(\tau)\right\| d \tau\right) .
$$

Further, for $\varepsilon \in\left(0, \frac{1}{2}\right)$ and $t \leq T$, with $C=C_{\varepsilon, T}$

$$
\int_{0}^{t}\left(\left\|u_{t}(\tau)\right\|_{\dot{H}^{2}}+\left\|u_{t t}(\tau)\right\|\right) d \tau \leq C\left(\left\|g_{0}\right\|_{H^{\varepsilon}}+\int_{0}^{t}\left\|f_{t}(\tau)\right\|_{H^{\varepsilon}} d \tau\right) .
$$

ProOF. We first note that, for $0<s \leq 1$,

$$
\left\|u_{t}(t)\right\|_{\dot{H}^{1+s}}=\left\|\Delta u_{t}(t)\right\|_{\dot{H}^{-1+s}} \leq\left\|u_{t t}(t)\right\|_{\dot{H}^{-1+s}}+\left\|f_{t}(t)\right\|_{\dot{H}^{-1+s}},
$$

so that it suffices to consider the second integrands on the left in (3.6) and (3.7). We now differentiate (3.4) to obtain

$$
u_{t}(t)=E(t) g_{0}+\int_{0}^{t} E(t-\tau) f_{t}(\tau) d \tau .
$$

By further differentiation this yields

$$
u_{t t}(t)=E^{\prime}(t) g_{0}+f_{t}(t)+\int_{0}^{t} E^{\prime}(t-\tau) f_{t}(\tau) d \tau .
$$


Let $\varepsilon=0$ if $s<1$ and $\varepsilon \in\left(0, \frac{1}{2}\right)$ if $s=1$. Then, by (3.3),

$$
\left\|E^{\prime}(t) g_{0}\right\|_{\dot{H}^{-1+s}}=\left\|E(t) g_{0}\right\|_{\dot{H}^{1+s}} \leq C t^{-\sigma}\left\|g_{0}\right\|_{\dot{H}^{\varepsilon}}, \quad \text { with } \sigma=(s+1-\varepsilon) / 2,
$$

and similarly for the integrand in (3.9). We conclude

$$
\left\|u_{t t}(t)\right\|_{\dot{H}^{-1+s}} \leq C\left(t^{-\sigma}\left\|g_{0}\right\|_{\dot{H}^{\varepsilon}}+\left\|f_{t}(t)\right\|_{\dot{H}^{\varepsilon}}+\int_{0}^{t}(t-\tau)^{-\sigma}\left\|f_{t}(\tau)\right\|_{\dot{H}^{\varepsilon}} d \tau\right)
$$

and hence after integration, since $\sigma=(s+1-\varepsilon) / 2<1$,

$$
\int_{0}^{t}\left\|u_{t t}(\tau)\right\|_{\dot{H}^{-1+s}} d \tau \leq C\left(1+T^{1-\sigma}\right)\left(\left\|g_{0}\right\|_{\dot{H}^{\varepsilon}}+\int_{0}^{t}\left\|f_{t}(\tau)\right\|_{\dot{H}^{\varepsilon}} d \tau\right) \text {, for } t \leq T \text {. }
$$

Since the norms in $\dot{H}^{\varepsilon}$ and $H^{\varepsilon}$ are equivalent, this completes the proof.

We remark that in (3.6) the $L_{2}$-norms on the right could have been replaced by fractional order negative norms. For simplicity of presentation we avoid using such norms in the statements of our results.

A second regularity result is as follows. Here, $g_{0} \in H_{0}^{1}$ involves a second compatibility condition at the boundary at initial time, viz., $\Delta v+f(0)=0$ on $\partial \Omega$.

LEMMA 3.2. We have for the solution of (1.1)

$$
\int_{0}^{t}\left\|u_{t}(\tau)\right\|_{H^{1}}^{2} d \tau \leq C\left(\left\|g_{0}\right\|^{2}+\int_{0}^{t}\left\|f_{t}(\tau)\right\|_{H^{-1}}^{2} d \tau\right)
$$

and

$$
\int_{0}^{t}\left(\left\|\Delta u_{t}(\tau)\right\|^{2}+\left\|u_{t t}(\tau)\right\|^{2}\right) d \tau \leq C\left(\left\|g_{0}\right\|_{H_{0}^{1}}^{2}+\int_{0}^{t}\left\|f_{t}(\tau)\right\|^{2} d \tau\right), \text { for } t \geq 0
$$

Proof. By differentiation of (1.1) and multiplication by $u_{t}$ in $L_{2}$ we obtain

$$
\frac{1}{2} \frac{d}{d t}\left\|u_{t}\right\|^{2}+\left\|\nabla u_{t}\right\|^{2}=\left(f_{t}, u_{t}\right) \leq C\left\|f_{t}\right\|_{H^{-1}}^{2}+\frac{1}{2}\left\|\nabla u_{t}\right\|^{2}
$$

and the first result hence follows by integration. Multiplication instead by $u_{t t}$ shows

$$
\left\|u_{t t}\right\|^{2}+\frac{1}{2} \frac{d}{d t}\left\|\nabla u_{t}\right\|^{2}=\left(f_{t}, u_{t t}\right) \leq \frac{1}{2}\left\|u_{t t}\right\|^{2}+\frac{1}{2}\left\|f_{t}\right\|^{2}
$$

which yields

$$
\int_{0}^{t}\left\|u_{t t}(\tau)\right\|^{2} d \tau \leq\left\|\nabla g_{0}\right\|^{2}+\int_{0}^{t}\left\|f_{t}(\tau)\right\|^{2} d \tau
$$

Since $\left\|\Delta u_{t}\right\| \leq\left\|u_{t t}\right\|+\left\|f_{t}\right\|$ the result follows.

We consider the semidiscrete problem (1.7) where for simplicity we take $v_{h}=$ $R_{h} v$, or, with $\Delta_{h}$ the discrete Laplacian and $P_{h}$ the orthogonal $L_{2}$-projection onto $S_{h}$,

$$
u_{h, t}-\Delta_{h} u_{h}=P_{h} f, \quad t>0, \quad \text { with } u_{h}(0)=R_{h} v
$$


The operator $-\Delta_{h}$ is a positive definite operator on $S_{h}$. The solution operator $E_{h}(t)=e^{\Delta_{h} t}$ of the homogeneous equation $(f=0)$ is again an analytic semigroup on $S_{h}$, uniformly in $h$. In particular,

$$
\left\|E_{h}(t) v_{h}\right\| \leq\left\|v_{h}\right\| \text { and }\left\|E_{h}^{\prime}(t) v_{h}\right\| \leq C t^{-1}\left\|v_{h}\right\| \text {, for } t>0 .
$$

We now show an error estimate for the solution of (3.10).

THEOREM 3.3. We have, with $C=C_{T}$,

$$
\left\|u_{h}(t)-u(t)\right\| \leq C h^{2 \beta}\left(\|\Delta v\|+\|f(0)\|+\int_{0}^{t}\left\|f_{t}(\tau)\right\| d \tau\right), \text { for } t \leq T .
$$

Proof. As in (1.8) we write $u_{h}(t)-u(t)=\theta(t)+\rho(t)$. For $\theta$ we have the representation (1.10), and hence, by the stability in $L_{2}$ of $E_{h}(t)$ and $P_{h}$,

$$
\left\|u_{h}(t)-u(t)\right\| \leq\|\rho(t)\|+\|\theta(t)\| \leq\|\rho(0)\|+2 \int_{0}^{t}\left\|\rho_{t}(\tau)\right\| d \tau
$$

Letting $\beta<s<1$, we have by the elliptic finite element estimate (2.13), together with (3.2),

$$
\left\|u_{h}(t)-u(t)\right\| \leq C h^{2 \beta}\left(\|v\|_{\dot{H}^{1+s}}+\int_{0}^{t}\left\|u_{t}(\tau)\right\|_{\dot{H}^{1+s}} d \tau\right), \text { for } t \geq 0 .
$$

Since $\|v\|_{\dot{H}^{1+s}} \leq C\|v\|_{\dot{H}^{2}}=C\|\Delta v\|$, the result stated now follows from (3.6) and (3.5).

We next show an $O\left(h^{\beta}\right)$ estimate for the gradient of the error.

THEOREM 3.4. We have for $t \leq T$, with $C=C_{T}$,

$\left\|\nabla\left(u_{h}(t)-u(t)\right)\right\| \leq C h^{\beta}\left(\|\Delta v\|+\|f(0)\|+\int_{0}^{t}\left\|f_{t}(\tau)\right\| d \tau+\left(\int_{0}^{t}\left\|f_{t}(\tau)\right\|_{H^{-1}}^{2} d \tau\right)^{1 / 2}\right)$.

Proof. Using Lemma 2.5 we find, with $\beta<s<1$,

$$
\|\nabla \rho(t)\| \leq C h^{\beta}\|u(t)\|_{\dot{H}^{1+s}} \leq C h^{\beta}\left(\|v\|_{\dot{H}^{1+s}}+\int_{0}^{t}\left\|u_{t}(\tau)\right\|_{\dot{H}^{1+s}} d \tau\right) .
$$

The right hand is bounded as desired by Lemma 3.1.

To bound $\nabla \theta(t)$ we choose $\chi=\theta_{t}$ in (1.9) to obtain

$$
\left\|\theta_{t}\right\|^{2}+\frac{1}{2} \frac{d}{d t}\|\nabla \theta\|^{2}=-\left(\rho_{t}, \theta_{t}\right) \leq \frac{1}{2}\left\|\rho_{t}\right\|^{2}+\frac{1}{2}\left\|\theta_{t}\right\|^{2} .
$$

Since $\theta(0)=0$ we have, using (2.14),

$$
\|\nabla \theta(t)\|^{2} \leq \int_{0}^{t}\left\|\rho_{t}(\tau)\right\|^{2} d \tau \leq C h^{2 \beta} \int_{0}^{t}\left\|u_{t}(\tau)\right\|_{H^{1}}^{2} d \tau, \text { for } t \geq 0 .
$$

The bound stated therefore follows by Lemma 3.2. 
The analysis above of $\nabla \theta$ also yields the following result about the "super"closeness of $u_{h}$ and $R_{h} u$.

LEMMA 3.5. We have

$$
\|\nabla \theta(t)\| \leq C h^{2 \beta}\left(\left\|g_{0}\right\|_{H_{0}^{1}}+\left(\int_{0}^{t}\left\|f_{t}(\tau)\right\|^{2} d \tau\right)^{1 / 2}\right), \text { for } t \geq 0 .
$$

Proof. Using (3.11) and (2.13) with $s=1$, we obtain

$$
\|\nabla \theta(t)\|^{2} \leq \int_{0}^{t}\left\|\rho_{t}(\tau)\right\|^{2} d \tau \leq C h^{4 \beta} \int_{0}^{t}\left\|\Delta u_{t}(\tau)\right\|^{2} d \tau
$$

where the last term is estimated by Lemma 3.2.

We shall now give an example of a nonsmooth data error estimate and demonstrate that, for the homogeneous parabolic equation, a $O\left(h^{2 \beta}\right)$ error estimate holds for the semidiscrete approximation for positive time even when the initial data are only assumed to be in $L_{2}$, provided the discrete initial data are appropriately chosen.

THEOREM 3.6. Let $u_{h}(t)$ and $u(t)$ be the solutions of (1.7) and (1.1) with $f=0$, and let $v_{h}=P_{h} v$. Then we have, for $\beta<s<1$, with $C=C_{s}$,

$$
\left\|u_{h}(t)-u(t)\right\| \leq C h^{2 \beta} t^{-(1+s) / 2}\|v\|, \text { for } t>0 .
$$

Proof. In [16, Chapter 3, formula (3.16)] the following inequality was shown for smooth $\partial \Omega$,

$$
\left\|u_{h}(t)-u(t)\right\| \leq C t^{-1} \sup _{\tau \leq t}\left(\tau^{2}\left\|\rho_{t}(\tau)\right\|+\tau\|\rho(\tau)\|+\|\tilde{\rho}(\tau)\|\right), \text { for } t>0,
$$

where $\tilde{\rho}(t)=\int_{0}^{t} \rho(\tau) d \tau$. However, for proving this, the smoothness of $\partial \Omega$ is not actually required. By (2.13) and (3.3), and using the definition of $\dot{H}^{-1+s}$ we easily obtain that

$$
\tau\|\rho(\tau)\| \leq C \tau h^{2 \beta}\|u(\tau)\|_{\dot{H}^{1+s}} \leq C h^{2 \beta} \tau^{(1-s) / 2}\|v\| .
$$

Hence, since $s<1$, we find

$$
\|\tilde{\rho}(\tau)\| \leq \int_{0}^{\tau}\|\rho(\eta)\| d \eta \leq C h^{2 \beta} \int_{0}^{\tau} \eta^{(-1-s) / 2}\|v\| d \eta \leq C h^{2 \beta} \tau^{(1-s) / 2}\|v\| .
$$

In the same way,

$$
\tau^{2}\left\|\rho_{t}(\tau)\right\| \leq C h^{2 \beta} \tau^{2}\left\|u_{t}(\tau)\right\|_{\dot{H}^{1+s}} \leq C h^{2 \beta} \tau^{(1-s) / 2}\|v\| .
$$

Together these inequalities complete the proof.

We finally show that the optimal order error bounds for the elliptic problem in Lemma 2.9 obtained by refinements towards the nonconvex corner can be carried over to the parabolic problem. 
THEOREM 3.7. Assume that the triangulations underlying the $S_{h}$ are refined as in Lemma 2.9. We then have, for $t \leq T$ and any $\varepsilon \in\left(0, \frac{1}{2}\right)$, with $C=C_{\varepsilon, T}$,

$$
\left\|u_{h}(t)-u(t)\right\| \leq C h^{2}\left(\|\Delta v\|+\left\|g_{0}\right\|_{H^{\varepsilon}}+\int_{0}^{t}\left\|f_{t}(\tau)\right\|_{H^{\varepsilon}} d \tau\right)
$$

and

$\left\|\nabla\left(u_{h}(t)-u(t)\right)\right\| \leq C h\left(\|\Delta v\|+\left\|g_{0}\right\|_{H^{\varepsilon}}+\int_{0}^{t}\left\|f_{t}(\tau)\right\|_{H^{\varepsilon}} d \tau+\left(\int_{0}^{t}\left\|f_{t}(\tau)\right\|_{H^{-1}}^{2} d \tau\right)^{1 / 2}\right)$.

Proof. We use Lemma 2.9 with $s=1$. We first have

$$
\|\rho(t)\|+h\|\nabla \rho(t)\| \leq C h^{2}\|\Delta u(t)\| \leq C h^{2}\left(\|\Delta v\|+\int_{0}^{t}\left\|\Delta u_{t}(\tau)\right\| d \tau\right) .
$$

The right hand side is now bounded by (3.7). For $\theta$ we have in a standard way, choosing $\chi=\theta$ in (1.9),

$$
\|\theta(t)\| \leq \int_{0}^{t}\left\|\rho_{t}(\tau)\right\| d \tau \leq C h^{2} \int_{0}^{t}\left\|\Delta u_{t}(\tau)\right\| d \tau, \text { for } t \geq 0
$$

where the right hand side is bounded as above. For the gradient of $\theta$ we have by the first inequality of (3.11),

$$
\|\nabla \theta(t)\|^{2} \leq \int_{0}^{t}\left\|\rho_{t}(\tau)\right\|^{2} d \tau \leq C h^{2} \int_{0}^{t}\left\|u_{t}(\tau)\right\|_{H^{1}}^{2} d \tau, \text { for } t \geq 0
$$

which is again bounded as desired by Lemma 3.2.

\section{Maximum-norm estimates.}

We now turn to maximum-norm error estimates for semidiscrete finite element approximations of (1.1), extending such estimates for the stationary elliptic problem. In the same way as in the discussion in Section 2, we shall be using two different approaches. In the first the analysis takes place within the framework of the Banach space $\mathcal{C}(\bar{\Omega})$ and assumes globally quasiuniform triangulations, and in the second the arguments are based on using the discrete Sobolev inequality, under weaker assumptions on the triangulations, together with $L_{2}$-bounds for the gradient of the $\theta$-part of the error.

We begin the first approach by showing a resolvent estimate for the discrete Laplacian $\Delta_{h}$, in the case of globally quasiuniform triangulations, and an associated stability and smoothing property for the solution operator of the homogeneous semidiscrete equation.

We recall that, in the case of a domain $\Omega$ with smooth boundary, the operator $\Delta$, with a suitably defined domain, satisfies the resolvent estimate

$$
\left\|(z I-\Delta)^{-1} v\right\|_{\mathcal{C}} \leq \frac{C}{1+|z|}\|v\|_{\mathcal{C}}, \quad \forall v \in \mathcal{C}_{0}(\bar{\Omega}), z \in \Sigma_{\alpha}=\{z:|\arg z|<\alpha\},
$$


for any $\alpha \in\left(\frac{1}{2} \pi, \pi\right)$, and hence generates an analytic semigroup $E(t)=e^{\Delta t}$ on $\mathcal{C}_{0}(\bar{\Omega})$, see Stewart [15]. It is not known to us if this holds also for a polygonal domain. However, the corresponding resolvent estimate is valid in $L_{p}$, for $1<$ $p<\infty$, as follows from the proof of Theorem 3.6 of Chapter 7 in [10].

The technique of proof of the following discrete resolvent estimate is based on [13]. For a domain with smooth boundary such a result was shown in [4] without the logarithmic factor $\ell_{h}$ (cf. (2.15)).

THEOREM 4.1. Assume that the family of triangulations underlying the $S_{h}$ is globally quasiuniform. Then, for any $\alpha \in\left(\frac{1}{2} \pi, \pi\right)$ and $h$ small, we have, with $C=C_{\alpha}$,

$$
\left\|\left(z I-\Delta_{h}\right)^{-1} v_{h}\right\|_{\mathcal{C}} \leq \frac{C \ell_{h}}{1+|z|}\left\|v_{h}\right\|_{\mathcal{C}}, \quad \forall v_{h} \in S_{h}, z \in \Sigma_{\alpha}
$$

Proof. For any $x \in \Omega$, let $\delta_{h}^{x} \in S_{h}$ be the discrete delta-function defined by

$$
\left(\chi, \delta_{h}^{x}\right)=\chi(x), \quad \forall \chi \in S_{h},
$$

and let $\omega_{h}^{x}$ be the modified distance function

$$
\omega_{h}^{x}(y)=\left(|y-x|^{2}+h^{2}\right)^{1 / 2}
$$

Here $\delta_{h}^{x}$ is concentrated near $x$ in the sense that one can show as in [16, Lemma 5.3], that, with $C$ independent of $x \in \Omega$ and $h$,

$$
\left\|\omega_{h}^{x} \delta_{h}^{x}\right\| \leq C, \text { for } x \in \Omega
$$

We now introduce the discrete Green's function

$$
G_{h}^{z ; x}=\left(z I-\Delta_{h}\right)^{-1} \delta_{h}^{x} \in S_{h},
$$

and note that, for $v_{h} \in S_{h}$,

$$
\left(z I-\Delta_{h}\right)^{-1} v_{h}(x)=\left(\left(z I-\Delta_{h}\right)^{-1} v_{h}, \delta_{h}^{x}\right)=\left(v_{h}, G_{h}^{\bar{z} ; x}\right) .
$$

To show the theorem we therefore want to show, with $C=C_{\alpha}$,

$$
\left\|G_{h}^{z ; x}\right\|_{L_{1}} \leq \frac{C \ell_{h}}{1+|z|}, \quad \forall x \in \Omega, z \in \Sigma_{\alpha} .
$$

Setting $\omega=\omega_{h}^{x}, G=G_{h}^{z ; x}$, and noting that $\left\|\omega^{-1}\right\| \leq C \ell_{h}^{1 / 2}$, we have

$$
\|G\|_{L_{1}} \leq\left\|\omega^{-1}\right\|\|\omega G\| \leq C \ell_{h}^{1 / 2}\|\omega G\|
$$

so that it suffices to show

$$
\|\omega G\| \leq \frac{C \ell_{h}^{1 / 2}}{1+|z|}, \quad \forall x \in \Omega, z \in \Sigma_{\alpha} .
$$


We consider the identity

$$
z\|\omega G\|^{2}+\|\omega \nabla G\|^{2}=z\left(G, \omega^{2} G\right)+\left(\nabla G, \nabla\left(\omega^{2} G\right)\right)-2(\nabla G, \omega G \nabla \omega) .
$$

Note that, with $\delta=\delta_{h}^{x}$,

$$
z(G, \chi)+(\nabla G, \nabla \chi)=\left(\left(z I-\Delta_{h}\right) G, \chi\right)=(\delta, \chi), \quad \forall \chi \in S_{h}, z \in \Sigma_{\alpha}
$$

Thus, subtracting this equation from the previous, and choosing $\chi=P_{h}\left(\omega^{2} G\right)$, where $P_{h}$ is the $L_{2}$-projection onto $S_{h}$, we find

$$
\begin{aligned}
z\|\omega G\|^{2}+\|\omega \nabla G\|^{2} & =z\left(G, \omega^{2} G-P_{h}\left(\omega^{2} G\right)\right)+\left(\nabla G, \nabla\left(\omega^{2} G-P_{h}\left(\omega^{2} G\right)\right)\right) \\
& -2(\nabla G, \omega G \nabla \omega)+\left(\delta, P_{h}\left(\omega^{2} G\right)\right)=\sum_{j=1}^{4} I_{j} .
\end{aligned}
$$

Obviously, $I_{1}=0$, since $G \in S_{h}$. To bound $I_{2}$ we need the following fact associated with superapproximation, cf. [16, Lemma 5.2], namely

$$
\left\|\nabla\left(\omega^{2} \chi-P_{h}\left(\omega^{2} \chi\right)\right)\right\| \leq C h(\|\chi\|+\|\omega \nabla \chi\|), \quad \forall \chi \in S_{h} .
$$

Using this, and also an inverse estimate, we find since $G \in S_{h}$, for any $\mu>0$,

$\left|I_{2}\right| \leq C h\|\nabla G\|(\|G\|+\|\omega \nabla G\|) \leq C\left(\|G\|^{2}+\|G\|\|\omega \nabla G\|\right) \leq \frac{\mu}{2}\|\omega \nabla G\|^{2}+C\|G\|^{2}$.

Also, since $\nabla \omega$ is bounded,

$$
\left|I_{3}\right| \leq C\|G\|\|\omega \nabla G\| \leq \frac{\mu}{2}\|\omega \nabla G\|^{2}+C\|G\|^{2},
$$

and for $I_{4}$ we have, using (4.1),

$$
\left|I_{4}\right|=\left|\left(\delta, \omega^{2} G\right)\right|=|(\omega \delta, \omega G)| \leq C\|\omega G\| .
$$

Altogether, after taking real and imaginary parts,

$$
\begin{aligned}
\operatorname{Re} z\|\omega G\|^{2}+\|\omega \nabla G\|^{2} & \leq \mu\|\omega \nabla G\|^{2}+C\left(\|G\|^{2}+\|\omega G\|\right), \\
|\operatorname{Im} z|\|\omega G\|^{2} & \leq \mu\|\omega \nabla G\|^{2}+C\left(\|G\|^{2}+\|\omega G\|\right) .
\end{aligned}
$$

Thus for any $\varepsilon>0$, after taking $\mu<\varepsilon /(2(1+\varepsilon))$ and kicking back $\|\omega \nabla G\|$,

$$
(|\operatorname{Im} z|+\varepsilon \operatorname{Re} z)\|\omega G\|^{2}+\frac{1}{2} \varepsilon\|\omega \nabla G\|^{2} \leq C\left(\|G\|^{2}+\|\omega G\|\right), \quad \text { with } C=C_{\varepsilon} .
$$

Choosing, e.g., $\varepsilon=\tan ((\pi-\alpha) / 2)$, we see that

$$
|\operatorname{Im} z|+\varepsilon \operatorname{Re} z \geq c|z|, \text { for } z \in \Sigma_{\alpha}, \quad \text { with } c>0 .
$$

Hence, with $C=C_{\alpha}$,

$$
|z|\|\omega G\|^{2}+\|\omega \nabla G\|^{2} \leq C\left(\|G\|^{2}+\|\omega G\|\right), \text { for } z \in \Sigma_{\alpha},
$$


and since

$$
C\|\omega G\| \leq \frac{1}{2}|z|\|\omega G\|^{2}+\frac{1}{2} C^{2}|z|^{-1},
$$

we obtain, with a new $C=C_{\alpha}$,

$$
|z|\|\omega G\|^{2}+\|\omega \nabla G\|^{2} \leq C\left(\|G\|^{2}+|z|^{-1}\right), \text { for } z \in \Sigma_{\alpha}
$$

To bound $\|G\|^{2}$, we note that

$$
z\|G\|^{2}+\|\nabla G\|^{2}=(\delta, G)=\bar{G}(x),
$$

and taking again real and imaginary parts, as above,

$$
|z|\|G\|^{2}+\|\nabla G\|^{2} \leq C|G(x)| \leq C \ell_{h}^{1 / 2}\|\nabla G\| \leq \frac{1}{2}\|\nabla G\|^{2}+C \ell_{h}, \text { for } z \in \Sigma_{\alpha},
$$

and hence

$$
|z|\|G\|^{2}+\|\nabla G\|^{2} \leq C \ell_{h}, \text { for } z \in \Sigma_{\alpha}, x \in \Omega
$$

Together (4.3) and (4.4) show, with $C=C_{\alpha}$,

$$
\|\omega G\| \leq C \ell_{h}^{1 / 2}|z|^{-1}, \text { for } z \in \Sigma_{\alpha}, x \in \Omega,
$$

which concludes the proof of (4.2) for $|z| \geq 1$, say. Since also, using (4.4) in the second step,

$$
\|\omega G\| \leq C\|\nabla G\| \leq C \ell_{h}^{1 / 2} \leq 2 C \frac{\ell_{h}^{1 / 2}}{1+|z|}, \text { for } z \in \Sigma_{\alpha},|z| \leq 1,
$$

the proof is complete.

As a consequence of the above discrete resolvent estimate the following result obtains in a standard way, cf. [10, Section 2.5].

THEOREM 4.2. The discrete Laplacian $\Delta_{h}$ generates an analytic semigroup $E_{h}(t)$ on $S_{h}$, equipped with the maximum-norm, defined for any $\alpha \in\left(\frac{1}{2} \pi, \pi\right)$ by

$$
E_{h}(t)=e^{t \Delta_{h}}=\frac{1}{2 \pi i} \int_{\partial \Sigma_{\alpha}} e^{t z}\left(z I-\Delta_{h}\right)^{-1} d z, \text { for } t>0
$$

with $E_{h}(0)=I$, and we have, with $C$ independent of $h$,

$$
\left\|E_{h}(t) v_{h}\right\|_{\mathcal{C}}+t\left\|E_{h}^{\prime}(t) v_{h}\right\|_{\mathcal{C}} \leq C \ell_{h}\left\|v_{h}\right\|_{\mathcal{C}}, \text { for } v_{h} \in S_{h}, t \geq 0 .
$$

We now show the following error estimate, with $g_{0}$ defined in (3.5).

THEOREM 4.3. Assume that the family of triangulations underlying the $S_{h}$ is quasiuniform. Then we have, for the solutions of (3.10) and (1.1), with $C=C_{T}$,

$$
\left\|u_{h}(t)-u(t)\right\|_{\mathcal{C}} \leq C h^{\beta} \ell_{h}^{2}\left(\|v\|_{C^{\beta}}+\left\|g_{0}\right\|+\int_{0}^{t}\left\|f_{t}(\tau)\right\| d \tau\right), \text { for } t \leq T .
$$


Proof. With our standard notation we have, using Lemma 2.6,

$$
\|\rho(t)\|_{\mathcal{C}} \leq C h^{\beta} \ell_{h}\|u(t)\|_{\mathcal{C}^{\beta}} \leq C h^{\beta} \ell_{h}\left(\|v\|_{\mathcal{C}^{\beta}}+\int_{0}^{t}\left\|u_{t}(\tau)\right\|_{\mathcal{C}^{\beta}} d \tau\right), \text { for } t \geq 0 .
$$

As before we use the representation (1.10) for $\theta(t)$. Using the stability result of Theorem 4.2 for $E_{h}(t)$ and the stability of $P_{h}$ in $\mathcal{C}$ (see, e.g. [16, Lemma 5.1]), we obtain

$$
\|\theta(t)\|_{\mathcal{C}} \leq C \ell_{h} \int_{0}^{t}\left\|\rho_{t}(\tau)\right\|_{\mathcal{C}} d \tau \leq C h^{\beta} \ell_{h}^{2} \int_{0}^{t}\left\|u_{t}(\tau)\right\|_{\mathcal{C}^{\beta}} d \tau, \text { for } t \geq 0
$$

By Lemmas 2.4 and 3.1 we find, with $\beta<s<1$, for $t \leq T$,

$$
\int_{0}^{t}\left\|u_{t}(\tau)\right\|_{\mathcal{C}^{\beta}} d \tau \leq C \int_{0}^{t}\left\|u_{t}(\tau)\right\|_{\dot{H}^{1+s}} d \tau \leq C_{T}\left(\left\|g_{0}\right\|+\int_{0}^{t}\left\|f_{t}(\tau)\right\| d \tau\right) .
$$

Together these estimates show the theorem.

We now turn to the second approach and derive a maximum-norm error estimate by using the error estimate for the gradient in $L_{2}$, together with the two-dimensional discrete Sobolev inequality. As in Lemma 2.7 this will be done under a weaker assumption on the triangulations than the one used in the first approach above.

THEOREM 4.4. Assume that the family of triangulations underlying $S_{h}$ is such that $h_{\text {min }} \geq C h^{\gamma}$ for some $\gamma>0$. Then, for any $s$ with $0 \leq s<\beta$, we have, for the solutions of (3.10) and (1.1), with $C=C_{s, T}$, for $t \leq T$,

$$
\left\|u_{h}(t)-u(t)\right\|_{\mathcal{C}} \leq C h^{s}\left(\|\Delta v\|+\|f(0)\|+\int_{0}^{t}\left\|f_{t}(\tau)\right\| d \tau+\left(\int_{0}^{t}\left\|f_{t}(\tau)\right\|_{H^{-1}}^{2} d \tau\right)^{1 / 2}\right) .
$$

Proof. We have by Lemma 2.7, with $s_{1} \in(s, \beta)$,

$$
\|\rho(t)\|_{\mathcal{C}} \leq C h^{s}\|\Delta u(t)\|_{H^{-1+s_{1}}} \leq C h^{s}\|u(t)\|_{\dot{H}^{1+s_{1}}} .
$$

Here, for $t \geq 0$,

$$
\|u(t)\|_{\dot{H}^{1+s_{1}}} \leq C\left(\|v\|_{\dot{H}^{1+s_{1}}}+\int_{0}^{t}\left\|u_{t}(\tau)\right\|_{\dot{H}^{1+s_{1}}} d \tau\right)
$$

which is bounded as desired by (3.6).

Using (2.16) together with (3.11) and Lemma 3.2 we have

$\|\theta(t)\|_{\mathcal{C}} \leq C \ell_{h}^{1 / 2}\|\nabla \theta(t)\| \leq C \ell_{h}^{1 / 2} h^{\beta}\left(\left\|g_{0}\right\|+\left(\int_{0}^{t}\left\|f_{t}(\tau)\right\|_{H^{-1}}^{2} d \tau\right)^{1 / 2}\right)$, for $t \geq 0$

Together these estimates show the result stated.

We now derive an estimate away from the corners. 
THEOREM 4.5. Let $\Omega_{0} \subset \Omega_{1} \subset \Omega$ be such that $\Omega_{1}$ does not contain any corner of $\Omega$ and the distance between $\partial \Omega_{1} \cap \Omega$ and $\partial \Omega_{0} \cap \Omega$ is positive. Assume that the triangulations associated with $S_{h}$ are quasiuniform in $\Omega_{1}$. Then we have, for $\beta<s<1, t \leq T$, with $C=C_{s, T}$,

$$
\begin{aligned}
\left\|u_{h}(t)-u(t)\right\|_{\mathcal{C}\left(\Omega_{0}\right)} \leq C h^{2 \beta} \ell_{h}^{1 / 2}( & \|u(t)\|_{\mathcal{C}^{2 s}\left(\Omega_{1}\right)}+\|\Delta v\|+\left\|g_{0}\right\|_{H_{0}^{1}} \\
& \left.+\left(\int_{0}^{t}\left\|f_{t}(\tau)\right\|^{2} d \tau\right)^{1 / 2}\right), \text { for } t \leq T .
\end{aligned}
$$

Proof. By Lemmas 2.8 and 3.1 we have, with $\beta<s<1$,

$$
\begin{aligned}
\|\rho(t)\|_{\mathcal{C}\left(\Omega_{0}\right)} & \leq C h^{2 \beta}\left(\|u(t)\|_{\mathcal{C}^{2 s}\left(\Omega_{1}\right)}+\|u(t)\|_{\dot{H}^{1+s}}\right) \\
& \leq C h^{2 \beta}\left(\|u(t)\|_{\mathcal{C}^{2 s}\left(\Omega_{1}\right)}+\|\Delta v\|+\left\|g_{0}\right\|+\int_{0}^{t}\left\|f_{t}(\tau)\right\| d \tau\right) \text {, for } t \leq T .
\end{aligned}
$$

Further, using the supercloseness result of Lemma 3.5,

$$
\|\theta(t)\|_{\mathcal{C}} \leq C \ell_{h}^{1 / 2}\|\nabla \theta(t)\| \leq C h^{2 \beta} \ell_{h}^{1 / 2}\left(\left\|g_{0}\right\|_{H_{0}^{1}}+\left(\int_{0}^{t}\left\|f_{t}(\tau)\right\|^{2} d \tau\right)^{1 / 2}\right), \text { for } t \geq 0 .
$$

Together these estimates show the error bound stated.

We also have the following result showing almost $O\left(h^{2}\right)$ convergence in the presence of appropriate refinements.

THEOREM 4.6. Let the triangulations underlying the $S_{h}$ be refined as in Lemma 2.10. Then, for any $s \in[0,2)$ and $t \geq 0$, we have, with $C=C_{s}$,

$\left\|u_{h}(t)-u(t)\right\|_{\mathcal{C}} \leq C h^{s}\left(\left\|g_{0}\right\|_{H_{0}^{1}}+\|f(0)\|_{\mathcal{C}}+\int_{0}^{t}\left\|f_{t}(\tau)\right\|_{\mathcal{C}} d \tau+\left(\int_{0}^{t}\left\|f_{t}(\tau)\right\|^{2} d \tau\right)^{1 / 2}\right)$.

Proof. To bound $\rho(t)$ we use Lemma 2.10 and (3.8), together with the fact that $E(t)$ is a contraction in $L_{p}$, to obtain, for $p$ sufficiently large, with $C=C_{s, p}$,

$$
\begin{aligned}
\|\rho(t)\|_{\mathcal{C}} & \leq C h^{s}\|\Delta u(t)\|_{L_{p}} \leq C h^{s}\left(\|f(t)\|_{L_{p}}+\left\|u_{t}(t)\right\|_{L_{p}}\right) \\
& \leq C h^{s}\left(\|f(0)\|_{L_{p}}+\left\|g_{0}\right\|_{L_{p}}+\int_{0}^{t}\left\|f_{t}(\tau)\right\|_{L_{p}} d \tau\right) \\
& \leq C h^{s}\left(\|f(0)\|_{\mathcal{C}}+\left\|\nabla g_{0}\right\|+\int_{0}^{t}\left\|f_{t}(\tau)\right\|_{\mathcal{C}} d \tau\right) .
\end{aligned}
$$

For $\theta(t)$ we first derive a superconvergent order estimate for $\nabla \theta(t)$ as in (3.12), now with the $L_{2}$ error estimate of Lemma 2.9 (valid since the refinement is now stronger than there), namely

$$
\|\nabla \theta(t)\|^{2} \leq \int_{0}^{t}\left\|\rho_{t}(\tau)\right\|^{2} d \tau \leq C h^{4} \int_{0}^{t}\left\|\Delta u_{t}(\tau)\right\|^{2} d \tau
$$

Using (2.16), and Lemma 3.2 to bound the last integral, we have

$$
\|\theta(t)\|_{\mathcal{C}} \leq C \ell_{h}^{1 / 2}\|\nabla \theta(t)\| \leq C \ell_{h}^{1 / 2} h^{2}\left(\left\|\nabla g_{0}\right\|+\left(\int_{0}^{t}\left\|f_{t}(\tau)\right\|^{2} d \tau\right)^{1 / 2}\right),
$$

which is bounded as stated for any $s \in[0,2)$. This completes the proof. 


\section{Fully discrete methods.}

As examples for fully discrete methods we will show some error estimates for the application of the Backward Euler and the Crank-Nicolson methods to the discretization in time of the spatially semidiscrete problem (1.7). Letting $k$ denote the constant time step, $U^{n}$ the approximation in $S_{h}$ of the exact solution $u(t)$ of (1.1) at $t=t_{n}=n k$, and setting $\bar{\partial} U^{n}=\left(U^{n}-U^{n-1}\right) / k$, we consider first the Backward Euler method

$$
\begin{aligned}
\left(\bar{\partial} U^{n}, \chi\right)+\left(\nabla U^{n}, \nabla \chi\right) & =\left(f^{n}, \chi\right), \quad \forall \chi \in S_{h}, n \geq 1, \\
U^{0} & =v_{h}=R_{h} v .
\end{aligned}
$$

We first show the following error estimate in $L_{2}-$ norm, with $g_{0}$ defined in (3.5).

THEOREM 5.1. Let $U^{n}$ and $u\left(t_{n}\right)$ be the solutions of (5.1) and (1.1), respectively, and let $\varepsilon \in\left(0, \frac{1}{2}\right)$. Then we have, with $C=C_{\varepsilon, T}$,

$$
\left\|U^{n}-u\left(t_{n}\right)\right\| \leq C\left(h^{2 \beta}+k\right)\left(\|\Delta v\|+\left\|g_{0}\right\|_{H^{\varepsilon}}+\int_{0}^{t_{n}}\left\|f_{t}(\tau)\right\|_{H^{\varepsilon}} d \tau\right), \text { for } t_{n} \leq T
$$

Proof. Analogously to (1.8) we write

$$
U^{n}-u\left(t_{n}\right)=\left(U^{n}-R_{h} u\left(t_{n}\right)\right)+\left(R_{h} u\left(t_{n}\right)-u\left(t_{n}\right)\right)=\theta^{n}+\rho^{n} .
$$

Here $\rho^{n}$ is bounded as desired as in the proof of Theorem 3.3. To bound $\theta^{n}$ we note that

$$
\left(\bar{\partial} \theta^{n}, \chi\right)+\left(\nabla \theta^{n}, \nabla \chi\right)=-\left(\omega^{n}, \chi\right), \quad \forall \chi \in S_{h}
$$

where

$$
\omega^{n}=\omega_{1}^{n}+\omega_{2}^{n}=\left(R_{h}-I\right) \bar{\partial} u\left(t_{n}\right)+\left(\bar{\partial} u\left(t_{n}\right)-u_{t}\left(t_{n}\right)\right) .
$$

Choosing $\chi=\theta^{n}$ in (5.3) we obtain in a standard fashion

$$
\left\|\theta^{n}\right\| \leq\left\|\theta^{n-1}\right\|+k\left\|\omega^{n}\right\|, \text { for } n \geq 1,
$$

and hence, since $\theta^{0}=0$,

$$
\left\|\theta^{n}\right\| \leq k \sum_{j=1}^{n}\left\|\omega_{1}^{j}\right\|+k \sum_{j=1}^{n}\left\|\omega_{2}^{j}\right\|=I+I I .
$$

Here $k \omega_{1}^{j}=\int_{t_{j-1}}^{t_{j}} \rho_{t}(\tau) d \tau$, and hence, as in Theorem 3.3,

$$
I \leq \int_{0}^{t_{n}}\left\|\rho_{t}(\tau)\right\| d \tau \leq C_{T} h^{2 \beta}\left(\left\|g_{0}\right\|+\int_{0}^{t_{n}}\left\|f_{t}(\tau)\right\| d \tau\right), \text { for } t_{n} \leq T .
$$

Further, using the second part of Lemma 3.1, we find, for any $\varepsilon>0$,

$$
I I \leq C k \int_{0}^{t_{n}}\left\|u_{t t}(\tau)\right\| d \tau \leq C_{\varepsilon} k\left(\left\|g_{0}\right\|_{H^{\varepsilon}}+\int_{0}^{t_{n}}\left\|f_{t}(\tau)\right\|_{H^{\varepsilon}} d \tau\right) .
$$


Together these estimates complete the proof.

Next, we will show the following estimate for the gradient of the error.

THEOREM 5.2. Let $U^{n}$ and $u\left(t_{n}\right)$ be the solutions of (5.1) and (1.1), respectively. Then we have, with $C=C_{T}$,

$\left\|\nabla\left(U^{n}-u\left(t_{n}\right)\right)\right\| \leq C\left(h^{\beta}+k\right)\left(\|\Delta v\|+\left\|g_{0}\right\|_{H_{0}^{1}}+\left(\int_{0}^{t_{n}}\left\|f_{t}(\tau)\right\|^{2} d \tau\right)^{1 / 2}\right)$, for $t_{n} \leq T$.

Proof. Here $\nabla \rho^{n}$ is bounded as desired by the proof of Theorem 3.4. Further, choosing $\chi=\bar{\partial} \theta^{n}$ in (5.3), since $2\left(\nabla \theta^{n}, \nabla \bar{\partial} \theta^{n}\right)=\bar{\partial}\left\|\nabla \theta^{n}\right\|^{2}+k\left\|\nabla \bar{\partial} \theta^{n}\right\|^{2}$ we find easily that $\bar{\partial}\left\|\nabla \theta^{n}\right\|^{2} \leq \frac{1}{2}\left\|\omega^{n}\right\|^{2}$, and hence

$$
\left\|\nabla \theta^{n}\right\|^{2} \leq k \sum_{j=1}^{n}\left\|\omega_{1}^{j}\right\|^{2}+k \sum_{j=1}^{n}\left\|\omega_{2}^{j}\right\|^{2}=I^{\prime}+I I^{\prime} .
$$

Here, using (3.11) and Lemma 3.2,

$$
I^{\prime} \leq \int_{0}^{t_{n}}\left\|\rho_{t}(\tau)\right\|^{2} d \tau \leq C h^{2 \beta}\left(\left\|g_{0}\right\|^{2}+\int_{0}^{t_{n}}\left\|f_{t}(\tau)\right\|_{H^{-1}}^{2} d \tau\right), \text { for } t_{n} \geq 0 .
$$

Further, by Lemma 3.2

$$
I I^{\prime} \leq C k^{2} \int_{0}^{t_{n}}\left\|u_{t t}(\tau)\right\|^{2} d \tau \leq C k^{2}\left(\left\|g_{0}\right\|_{H_{0}^{1}}^{2}+\int_{0}^{t_{n}}\left\|f_{t}(\tau)\right\|^{2} d \tau\right) \text {, for } t_{n} \geq 0 .
$$

Together these estimates complete the proof.

Also, we will show the following nonsmooth initial data estimate.

THEOREM 5.3. Let $U^{n}$ and $u\left(t_{n}\right)$ be the solutions of (5.1) and (1.1) with $f=0$, but with $v_{h}=P_{h} v$. Then we have, for $\beta<s<1$, with $C=C_{s, T}$,

$$
\left\|U^{n}-u\left(t_{n}\right)\right\| \leq C\left(h^{2 \beta} t_{n}^{-(1+s) / 2}+k t_{n}^{-1}\right)\|v\|, \text { for } t_{n} \leq T .
$$

Proof. In view of Theorem 3.6 it suffices to note that

$$
\left\|U^{n}-u_{h}\left(t_{n}\right)\right\| \leq C k t_{n}^{-1}\left\|P_{h} v\right\| \leq C k t_{n}^{-1}\|v\|, \text { for } t_{n} \leq T .
$$

The former inequality is a special case of, e.g., [16, Theorem 7.2].

We now demonstrate two maximum-norm error estimates, using the two approaches to such estimates used earlier in Sections 2 and 4. We first have the following.

THEOREM 5.4. Assume that the family of triangulations underlying the $S_{h}$ is globally quasiuniform. Let $U^{n}$ and $u\left(t_{n}\right)$ be the solutions of (5.1) and (1.1), respectively, and let $g_{1}=u_{t t}(0)=\Delta g_{0}+f_{t}(0)$. Then, with $C=C_{T}$, we have, for $t_{n} \leq T$,

$$
\left\|U^{n}-u\left(t_{n}\right)\right\|_{\mathcal{C}} \leq C\left(h^{\beta}+k\right) \ell_{h}^{2}\left(\|v\|_{\mathcal{C}^{\beta}}+\left\|g_{0}\right\|+\left\|g_{1}\right\|+\int_{0}^{t_{n}}\left(\left\|f_{t}(\tau)\right\|+\left\|f_{t t}(\tau)\right\|\right) d \tau\right) .
$$


Proof. The term $\rho^{n}$ is bounded as stated by (4.5) and (4.6). For $\theta^{n}$ we have, by $(5.3)$,

$$
\theta^{n}-k \Delta_{h} \theta^{n}=\theta^{n-1}-k P_{h} \omega^{n}
$$

or, with $E_{k h}=\left(I-k \Delta_{h}\right)^{-1}$, since $\theta^{0}=0$,

$$
\theta^{n}=E_{k h}\left(\theta^{n-1}-k P_{h} \omega^{n}\right)=-k \sum_{j=1}^{n} E_{k h}^{n+1-j} P_{h} \omega^{j} .
$$

By use of the resolvent estimate of Theorem 4.1, see, e.g., [16, Theorem 8.2], we have the stability bound

$$
\left\|E_{k h}^{m} P_{h} v\right\|_{\mathcal{C}} \leq C \ell_{h}\|v\|_{\mathcal{C}}, \text { for } m \geq 0,
$$

and hence, with the notation (5.4),

$$
\left\|\theta^{n}\right\|_{\mathcal{C}} \leq C k \ell_{h} \sum_{j=1}^{n}\left\|\omega_{1}^{j}\right\|_{\mathcal{C}}+C k \ell_{h} \sum_{j=1}^{n}\left\|\omega_{2}^{j}\right\|_{\mathcal{C}}=I^{\prime \prime}+I I^{\prime \prime} .
$$

Here, using Lemmas 2.6 and 2.4, we have, with $\beta<s<1$,

$$
I^{\prime \prime} \leq C h^{\beta} \ell_{h}^{2} \int_{0}^{t_{n}}\left\|u_{t}(\tau)\right\|_{\mathcal{C}^{\beta}} d \tau \leq C h^{\beta} \ell_{h}^{2} \int_{0}^{t_{n}}\left\|u_{t}(\tau)\right\|_{\dot{H}^{1+s}} d \tau
$$

which is bounded as desired by (3.6). Further, for any $\varepsilon>0$,

$$
I I^{\prime \prime} \leq C k \ell_{h} \int_{0}^{t_{n}}\left\|u_{t t}(\tau)\right\|_{\mathcal{C}} d \tau \leq C k \ell_{h} \int_{0}^{t_{n}}\left\|u_{t t}(\tau)\right\|_{\dot{H}^{1+\varepsilon}} d \tau .
$$

By (3.6), applied to $u_{t}$, we have the regularity estimate

$$
\int_{0}^{t_{n}}\left\|u_{t t}(\tau)\right\|_{\dot{H}^{1+\varepsilon}} d \tau \leq C_{T}\left(\left\|g_{1}\right\|+\int_{0}^{t_{n}}\left\|f_{t t}(\tau)\right\| d \tau\right), \text { for } t_{n} \leq T,
$$

which completes the proof.

By using the technique for estimating $\rho$ in the proof of Theorem 4.4, combined with the discrete Sobolev inequality (2.16) and the estimate for $\nabla \theta^{n}$ in the proof of Theorem 5.2, we have the following.

THEOREM 5.5. Assume the family of triangulations underlying $S_{h}$ is such that $h_{\text {min }} \geq C h^{\gamma}$ for some $\gamma>0$. Then, for any $s$ with $0 \leq s<\beta$, we have, for the solutions of (5.1) and (1.1), with $C=C_{s, T}$, for $t \leq T$,

$$
\left\|U^{n}-u\left(t_{n}\right)\right\|_{\mathcal{C}} \leq C\left(h^{s}+\ell_{h}^{1 / 2} k\right)\left(\|\Delta v\|+\left\|g_{0}\right\|_{H_{0}^{1}}+\left(\int_{0}^{t}\left\|f_{t}(\tau)\right\|^{2} d \tau\right)^{1 / 2}\right) .
$$

As a final example of a fully discrete method we will consider the CrankNicolson method for the discretization in time of the semidiscrete problem (1.7), 
combined with such refinement in space that yields an optimal order $O\left(h^{2}\right)$ error estimate in space. With the above notation, and setting $\widehat{U}^{n}=\frac{1}{2}\left(U^{n}+U^{n-1}\right)$, the Crank-Nicolson method is defined by

$$
\begin{aligned}
\left(\bar{\partial} U^{n}, \chi\right)+\left(\nabla \widehat{U}^{n}, \nabla \chi\right) & =\left(f\left(t_{n-\frac{1}{2}}\right), \chi\right), \quad \forall \chi \in S_{h}, n \geq 1, \\
U^{0} & =v_{h}=R_{h} v .
\end{aligned}
$$

We show the following error estimate.

THEOREM 5.6. Let $U^{n}$ and $u\left(t_{n}\right)$ be the solutions of (5.6) and (1.1), and let $\varepsilon \in\left(0, \frac{1}{2}\right)$. Assume that the triangulations are refined as in Lemma 2.9. Then we have, with $g_{1}$ as in Theorem 5.4, and $C=C_{\varepsilon, T}$,

$$
\begin{aligned}
\left\|U^{n}-u\left(t_{n}\right)\right\| \leq & C\left(h^{2}+k^{2}\right)\left(\|\Delta v\|+\left\|g_{0}\right\|_{H^{\varepsilon}}+\left\|g_{1}\right\|_{H^{\varepsilon}}\right. \\
& \left.+\int_{0}^{t_{n}}\left(\left\|f_{t}(\tau)\right\|_{H^{\varepsilon}}+\left\|f_{t t}(\tau)\right\|_{H^{\varepsilon}}\right) d \tau\right), \text { for } t_{n} \leq T .
\end{aligned}
$$

Proof. We again represent the error as in (5.2). For $\rho^{n}$ we have, as in the proof of Theorem 3.7,

$$
\left\|\rho^{n}\right\| \leq C h^{2}\left(\|\Delta v\|+\left\|g_{0}\right\|_{H^{\varepsilon}}+\int_{0}^{t_{n}}\left\|f_{t}(\tau)\right\|_{H^{\varepsilon}} d \tau\right) .
$$

To bound $\theta^{n}$ we note that this time

$$
\left(\bar{\partial} \theta^{n}, \chi\right)+\left(\nabla \widehat{\theta}^{n}, \nabla \chi\right)=-\left(\omega^{n}, \chi\right), \quad \forall \chi \in S_{h}, n \geq 1,
$$

where

$\omega^{n}=\left(R_{h}-I\right) \bar{\partial} u\left(t_{n}\right)+\left(\bar{\partial} u\left(t_{n}\right)-u_{t}\left(t_{n-\frac{1}{2}}\right)\right)-\Delta\left(u\left(t_{n-\frac{1}{2}}\right)-\widehat{u}\left(t_{n}\right)\right)=\omega_{1}^{n}+\omega_{2}^{n}+\omega_{3}^{n}$.

Choosing $\chi=\widehat{\theta}^{n}$ in (5.7) we obtain

$$
\left(\bar{\partial} \theta^{n}, \frac{1}{2}\left(\theta^{n}+\theta^{n-1}\right)\right) \leq \frac{1}{2}\left\|\omega^{n}\right\|\left(\left\|\theta^{n}\right\|+\left\|\theta^{n-1}\right\|\right),
$$

which gives

$$
\left\|\theta^{n}\right\|^{2}-\left\|\theta^{n-1}\right\|^{2} \leq k\left\|\omega^{n}\right\|\left(\left\|\theta^{n}\right\|+\left\|\theta^{n-1}\right\|\right), \text { for } n \geq 1,
$$

and, after cancellation of a common factor,

$$
\left\|\theta^{n}\right\| \leq\left\|\theta^{n-1}\right\|+k\left\|\omega^{n}\right\|, \text { for } n \geq 1 .
$$

Since $\theta^{0}=0$ this yields

$$
\left\|\theta^{n}\right\| \leq k \sum_{j=1}^{n}\left\|\omega_{1}^{j}\right\|+k \sum_{j=1}^{n}\left\|\omega_{2}^{j}\right\|+k \sum_{j=1}^{n}\left\|\omega_{3}^{j}\right\| .
$$


Here, as in (5.5) and the proof of Theorem 3.7,

$$
k \sum_{j=1}^{n}\left\|\omega_{1}^{j}\right\| \leq \int_{0}^{t_{n}}\left\|\rho_{t}(\tau)\right\| d \tau \leq C h^{2}\left(\left\|g_{0}\right\|_{H^{\varepsilon}}+\int_{0}^{t_{n}}\left\|f_{t}(\tau)\right\|_{H^{\varepsilon}} d \tau\right) \text {, for } t_{n} \leq T .
$$

Further, by Taylor expansion around $t_{n-\frac{1}{2}}$,

$$
k\left\|\omega_{2}^{j}\right\|=\left\|u\left(t_{j}\right)-u\left(t_{j-1}\right)-k u_{t}\left(t_{j-\frac{1}{2}}\right)\right\| \leq C k^{2} \int_{t_{j-1}}^{t_{j}}\left\|u_{t t t}(\tau)\right\| d \tau
$$

and similarly

$$
k\left\|\omega_{3}^{j}\right\|=\left\|\Delta\left(u\left(t_{j-\frac{1}{2}}\right)-\frac{1}{2}\left(u\left(t_{j}\right)-u\left(t_{j-1}\right)\right)\right)\right\| \leq C k^{2} \int_{t_{j-1}}^{t_{j}}\left\|\Delta u_{t t}(\tau)\right\| d \tau .
$$

Using once more the differential equation we get $\left\|\Delta u_{t t}\right\| \leq\left\|u_{t t t}\right\|+\left\|f_{t t}\right\|$. Thus,

$$
k \sum_{j=1}^{n}\left(\left\|\omega_{2}^{j}\right\|+\left\|\omega_{3}^{j}\right\|\right) \leq C k^{2} \int_{0}^{t_{n}}\left(\left\|u_{t t t}(\tau)\right\|+\left\|f_{t t}(\tau)\right\|\right) d \tau .
$$

Applying (3.7) to $u_{t t}$ we obtain

$$
\int_{0}^{t_{n}}\left\|u_{t t t}(\tau)\right\| d \tau \leq C_{\varepsilon}\left(\left\|g_{1}\right\|_{H^{\varepsilon}}+\int_{0}^{t_{n}}\left\|f_{t t}(\tau)\right\|_{H^{\varepsilon}} d \tau\right), \text { for } t_{n} \leq T,
$$

which bounds $\theta^{n}$ as desired. The proof is now complete.

Using techniques similar to the above one may also show an error bound in maximum-norm for the Crank-Nicolson method, with the appropriate mesh refinement as in Lemma 2.10, of order essentially $O\left(h^{2}+k^{2}\right)$.

\section{Appendix. Proof of Lemma 2.1.}

Proof. Recall that $H^{s}=\left[L_{2}, H^{1}\right]_{s, 2}$ and $\dot{H}^{s}=\left[L_{2}, H_{0}^{1}\right]_{s, 2}$. We shall show that

$$
\left[L_{2}, H^{1}\right]_{s, q}=\left[L_{2}, H_{0}^{1}\right]_{s, q}, \text { for } 0<s<\frac{1}{2}, q=\infty .
$$

Using this with $0<s_{1}<s<s_{2}<\frac{1}{2}$ and interpolating between $s_{1}, s_{2}$ it follows for any $q$, in particular for $q=2$. Since the opposite inclusion is trivial it now suffices to show

$$
B_{2}^{s, \infty}=\left[L_{2}, H^{1}\right]_{s, \infty} \subset\left[L_{2}, H_{0}^{1}\right]_{s, \infty}, \text { for } 0<s<\frac{1}{2} .
$$

Let $u \in\left[L_{2}, H^{1}\right]_{s, \infty}$. Then

$$
K\left(t, u ; L_{2}, H^{1}\right)=\inf _{v \in H^{1}}\left(\|u-v\|+t\|v\|_{H^{1}}\right) \leq U_{s} t^{s}, \quad \text { where } U_{s}=\|u\|_{B_{2}^{s, \infty}},
$$

so that there exists $v=v(t) \in H^{1}$ with

$$
\|u-v\| \leq C U_{s} t^{s} \quad \text { and }\|v\|_{H^{1}} \leq C U_{s} t^{s-1}
$$


We want to show that for some $w=w(t) \in H_{0}^{1}$ and some constant $C$,

$$
\|u-w\|+t\|w\|_{H^{1}} \leq C U_{s} t^{s} .
$$

It suffices to do this for $t \leq 1$, say, because for $t>1$ we may choose $w(t)=0$, in which case, by (A.1),

$$
\|u-w\|+t\|w\|_{H^{1}}=\|u\| \leq\|u-v\|+\|v\| \leq C U_{s} t^{s} \text {, for } t>1 .
$$

Let $\varphi_{t} \in C_{0}^{1}(\Omega)$ be such that $\varphi_{t}(x)=1$ in $\Omega_{t}$, the points in $\Omega$ with distance at least $t$ from $\partial \Omega$, and $\left|\nabla \varphi_{t}\right| \leq C / t$. We now choose $w(t)=\varphi_{t} v(t) \in H_{0}^{1}$. We have with $\omega_{t}=\Omega \backslash \Omega_{t}$,

$$
\begin{aligned}
\|u-w\|+t\|\nabla w\| & \leq\left\|\left(1-\varphi_{t}\right) u\right\|+\left\|\varphi_{t}(u-v)\right\|+t\left\|\nabla \varphi_{t} v\right\|+t\left\|\varphi_{t} \nabla v\right\| \\
& \leq C\left(\|u\|_{L_{2}\left(\omega_{t}\right)}+\|u-v\|+\|v\|_{L_{2}\left(\omega_{t}\right)}+t\|\nabla v\|\right) .
\end{aligned}
$$

In view of $(\mathrm{A} .1)$ and since $\|v\|_{L_{2}\left(\omega_{t}\right)} \leq\|u\|_{L_{2}\left(\omega_{t}\right)}+\|v-u\|$, it remains to show that

$$
\|u\|_{L_{2}\left(\omega_{t}\right)} \leq C t^{s} U_{s}=C t^{s}\|u\|_{B_{2}^{s, \infty}} .
$$

To prove this we note that, for any straight line $\gamma$ in $\Omega$, we have the trace inequality

$$
\|u\|_{L_{2}(\gamma)} \leq C\|u\|_{B_{2}^{1 / 2,1}} .
$$

For this we may first extend functions from $\Omega$ to $\mathbb{R}^{2}$ using [17, Theorem 4.2.3] and then [17, Theorem 2.9.3(c)]. Applying this to lines parallel to $\partial \Omega$ shows, after squaring and integrating,

$$
\|u\|_{L_{2}\left(\omega_{t}\right)} \leq C t^{1 / 2}\|u\|_{B_{2}^{1 / 2,1}}
$$

The desired result (A.2) now follows by interpolation between this and the trivial inequality $\|u\|_{L_{2}\left(\omega_{t}\right)} \leq\|u\|$.

\section{REFERENCES}

1. I. Babuška, Finite element method for domains with corners, Computing, 6 (1970), pp. 264-273.

2. C. Bacuta, J. H. Bramble, and J. E. Pasciak, New interpolation results and applications to finite element methods for elliptic boundary value problems, East-West J. Num. Math., 3 (2001), pp. 179-198.

3. C. Bacuta, J. H. Bramble, and J. Xu, Regularity estimates for elliptic boundary value problems in Besov spaces, Math. Comp., 72 (2003), pp. 1577-1595.

4. N. Bakaev, V. Thomée, and L. B. Wahlbin, Maximum-norm estimates for resolvents of elliptic finite element operators, Math. Comp., 72 (2003), pp. 1597-1610.

5. P. Grisvard, Caractérisation de quelques éspaces d'interpolation, Arch. Rat. Mech. Anal., 25 (1967), pp. 41-63.

6. P. Grisvard, Elliptic Problems in Nonsmooth Domains, Pitman, Massachusetts, 1985. 
7. P. Grisvard, Singularities in Boundary Value Problems, Masson, 1992.

8. B. R. Kellogg, Interpolation between subspaces of a Hilbert space, Technical note BN-719, Institute for Fluid Dynamics and Applied Mathematics, College Park, 1971.

9. V. G. Maz'ya and B. A. Plamenevskii, On the coefficients in the asymptotics of solutions of elliptic boundary value problems near the edge, Soviet Math. Doklady, 17 no. 4 (1976), pp. 970-974.

10. A. Pazy, Semigroups of Linear Operators and Applications to Partial Differential Equations, Springer-Verlag, New York, 1983.

11. G. Raugel, Résolution numérique par une méthode d'élements finis du problème de Dirichlet pour le laplacien dans un polygone, C.R. Acad. Sc. Paris, Série A, 286 (1977), pp. 791-794.

12. A. H. Schatz, A weak discrete maximum principle and stabiity of the finite element method in $L_{\infty}$ on plane polygonal domains. I, Math. Comp., 34 (1980), pp. 77-91.

13. A. H. Schatz, V. Thomée, and L. B. Wahlbin, Maximum norm stability and error estimates in parabolic finite element equations, Comm. Pure Appl. Math., 33 (1980), pp. 265-304.

14. A. H. Schatz and L. B. Wahlbin, Maximum norm estimates in the finite element method on plane polygonal domains, Part 2, Refinements, Math. Comp., 33 (1979), pp. 465-492.

15. B. Stewart, Generation of analytic semigroups by strongly elliptic operators, Trans. Amer. Math. Soc., 199 (1974), pp. 141-161.

16. V. Thomée, Galerkin Finite Element Methods for Parabolic Problems, SpringerVerlag, New York, 1997.

17. H. Triebel, Interpolation Theory, Function Spaces, Differential Operators, North Holland, 1978.

18. L. B. Wahlbin, On the sharpness of certain local estimates for $H_{0}^{1}$ projections into finite element spaces: Influence of a reentrant corner, Math. Comp., 42 (1984), pp. 1-8.

19. L. B. Wahlbin, Local Behavior in Finite Element Methods, Handbook of Numerical Analysis, vol. II, Finite element Methods (Part 1), P.G. Ciarlet and J.L. Lions, Eds, Elsevier, 1991, pp. 353-522.

20. M. F. Wheeler, A priori $L_{2}$ error estimates for Galerkin approximations to parabolic partial differential equations, SIAM J. Numer. Anal., 10 (1973), pp. 723-759.

21. J. -L. Zolesio, Interpolation d'éspaces de Sobolev avec conditions aux limites de type mélé, C. R. Acad. Sc. Paris, 285 (1977), pp. 621-624. 